\title{
7 Vermeidung problematischer Mediennutzungsmuster im Kindes- und Jugendalter
}

In den ersten Kapiteln dieser Arbeit konnte gezeigt werden, dass die Mediennutzung zwar einen wichtigen und vielfach funktionellen Beitrag im Leben von Kindern und Jugendlichen leistet, dass aber gleichzeitig von bestimmten Mediennutzungsmustern negative Wirkrisiken ausgehen. Im vorangegangenen Kapitel wurden diese Mediennutzungsmuster im Sinne eines Risikoverhaltens als problematische oder missbräuchliche Mediennutzung klassifiziert und näher beschrieben. Im Rahmen des folgenden Kapitels soll erörtert werden, unter welchen Bedingungen und mit welchem Instrumentarium es gelingen kann, medienbezogenes Risikoverhalten von Minderjährigen wirkungsvoll zu verhindern.

Hierbei gilt zu berücksichtigen, was über problematische Mediennutzung von Minderjährigen zusammengetragen wurde: Es wurde deutlich gemacht, dass Hinweise problematischer Mediennutzungsmuster bereits bei 7 bis 19 Prozent $^{114}$ der Kinder im Vorschulalter zu finden sind, dass problematische zeitliche und inhaltliche Mediennutzung bei Minderjährigen im Grundschul- und Jugendalter im (teilweise deutlich) zweistelligen Prozentbereich liegt, dass insbesondere bezüglich der Fernsehnutzungszeiten im späten Grundschulalter rund die Hälfte der Jungen und mehr als ein Drittel der Mädchen Mediennutzungszeiten aufweisen, die bereits negative Effekte auf die schulische Leistungsfähigkeit annehmen lassen (vgl. S. 144 - 147 in dieser Arbeit) und dass sich im Jugendalter inhaltlich und zeitlich problematische Mediennutzung insbesondere bei Jungen weiter verfestigt. Insofern sprechen allein diese Fakten dafür, medienbezogenem Risikoverhalten möglichst früh entgegenzuwirken.

1147 Prozent der Klein- und Vorschulkinder haben einen eigenen Fernseher im Zimmer, 19 Prozent schauen (fast) täglich alleine Fernsehen (vgl. S. 26 - 28 in dieser Arbeit). Zur Frage, ob diese Kennzahlen lediglich Risikoindikatoren oder aber eindeutige Indikatoren für tatsächlich vorhandene problematische Mediennutzung sind, vgl. die Diskussion auf S. 143). 


\subsection{Vorüberlegungen}

Aus der entwicklungspsychologischen Forschung und der psychologischen Gesundheitsforschung zum Risikoverhalten von Kindern und Jugendlichen ergeben sich weitere stützende Elemente für die Notwendigkeit möglichst früh einsetzender Maßnahmen gegen die Etablierung problematischer Mediennutzungsmuster im Kindesalter: Allgemein kann die Adoleszenz als eine Entwicklungsstufe gesehen werden, die als „window of vulnerability“ für gesundheitsschädigende Verhaltensweisen und allgemeiner Risikobereitschaft gelten (Seiffge-Krenke, 2008, S. 829). Das Gefühl der Invulnerabilität (,,personal fable"), die Unterschätzung der eigenen Gefährdung durch eigenes oder fremdes Handeln, die Höhergewichtung (angenehmer) unmittelbarer Konsequenzen vor möglichen (negativen) Spätfolgen sowie die Ausblendung oder Marginalisierung bewusster Risiken in konkreten Handlungskontexten gilt für die Adoleszenz als ebenso belegt wie die Tatsache, dass Risikoverhalten als Ausdruck von Autonomiebestrebungen gegenüber dem Elternhaus von Jugendlichen bewusst inszeniert wird. Als verstärkendes Element gilt ein das eigene Verhalten stützende Peermodell (ebenda). Insofern ist jugendliches Risikoverhalten deutlich ausgeprägter als das Risikoverhalten in der Kindheit oder in späteren Lebensphasen. Gleichzeitig sind Jugendliche nur schwer durch Präventions- beziehungsweise Interventionsmaßnahmen zu erreichen, zudem erweisen sich solche Maßnahmen im Jugendalter häufig als wenig effektiv (Jerusalem, 2003, S. 462 - 463; Roth, Rudert \& Petermann, 2003, S. 402). So ist nicht zu leugnen, dass medienbezogenes Risikoverhalten von Jugendlichen - analog zu den Erkenntnissen von Jerusalem - als funktional wahrgenommen wird für die Anerkennung im Freundeskreis, die Erweiterung des Erfahrungshorizonts, für die Kompensation von Selbstwertdefiziten, für die Belastungsregulation, für die Demonstration der Zugehörigkeit zu einer Subkultur sowie für den Nachweis von Autonomie und Erwachsensein (vgl. Jerusalem, 2003, S. 462). Es zeigt sich also auch vor diesem Hintergrund, dass Menschen im Jugendalter, in dem sie ein besonders hohes $\mathrm{Ma} ß$ an Risikoverhalten zeigen, schwer durch entsprechende Risikopräventionsprogramme zu erreichen sind, und dass Ansätze, die eine starke Ausprägung solcher Verhaltensformen im Jugendalter zu verhindern suchen, bereits vor Erreichen der Adoleszenz beginnen sollten.

Auch die Erkenntnisse der Mediennutzungsforschung zeigen, dass der Ausbildung problematischer Mediennutzungsmuster bereits frühzeitig begegnet werden sollte. So konnte im Rahmen verschiedener Längsschnittstudien nachgewiesen werden, dass Mediennutzungsmuster von der Kindheit bis ins Erwachsenenalter relativ stabil sind und dass die Wurzeln problematischer 
Mediennutzungsmuster in Adoleszenz und Erwachsenenalter überwiegend bereits in der Kindheit zu finden sind. Zwar ändert sich im Laufe der kindlichen Entwicklung und der damit einhergehenden zunehmenden Kompetenzen im Umgang mit (Schrift-)Sprache, narrativen Zusammenhängen und komplexen medialen Darstellungen die Breite der zur Verfügung stehenden Medien und Inhalte, eine Bindung an bestimmte Leitmedien, damit einhergehende Nutzungszeiten sowie grobe Genrepräferenzen bilden sich jedoch bereits frühzeitig heraus.

Insbesondere konnte dies für das Fernsehen gezeigt werden. So wiesen Zimmerman und Christakis (2005) Korrelationen der kindlichen Fernsehnutzungszeiten im Alter von unter drei Jahren, im Alter von drei bis 5 Jahren und im Alter von sechs Jahren von $r=0.47^{115}, r=0.60^{116}$ und $r=0.29^{117}$ nach (S. 621). Ennemoser und Schneider (2007) zeigten für die Spanne zwischen Vorschulalter und fünfter Klasse Korrelationen der Fernsehzeiten zu den unterschiedlichen Messzeitpunkten für Unterhaltungsfernsehen sowie für Bildungsfernsehen zwischen $r=0.36$ und $r=0.81$ (S. 356). Ähnliche Kennwerte berichten Koolstra et al. (1997) bezüglich der Gesamtfernsehzeit, Unterhaltungsfernsehnutzung und Bildungsfernsehnutzung für Kinder, deren Fernsehnutzung von der zweiten bis sechsten Klasse kontinuierlich gemessen wurde (S. 138). Rehbein (2010) zeigte mithilfe einer Retrospektivbefragung an jungen Erwachsenen, dass Fernsehnutzungszeiten wie auch Computerspielnutzungszeiten ab dem Grundschulalter bis in die Adoleszenz als recht stabil gelten können. Auch bezüglich problematischer Mediennutzung konnten Längsschnittstudien von Hopf et al. (2008, S. 84) sowie von Möller und Krahé (2009, S. 82) sowie von Wallenius und Punamäki (2008, S. 289) zeigen, dass die intraindividuelle Gewaltmedienexposition bezüglich Fernseh-/Videogewalt und/oder Computerspielgewalt im Alter von 10 bis 13 Jahren mit der Gewaltmedienexposition im Alter von 12 bis 16 Jahren auf mittlerem bis starkem Niveau korreliert. Obwohl dies keineswegs überraschend ist, zeigen auch diese Daten doch noch einmal eindringlich, dass Maßnahmen, die medienbezogenes Risikoverhalten von Minderjährigen zu verhindern suchen, möglichst früh einsetzen sollten.

Offen bleibt bis hierher, mit welchem Instrumentarium solche Maßnahmen arbeiten sollen, welche Akteure in diese Maßnahmen eingebunden werden

115 Fernsehnutzungszeiten im Alter von unter 3 Jahren korreliert mit Fernsehnutzungszeiten im Alter von 3 - 5 Jahren.

116 Fernsehnutzungszeiten im Alter von 3 - 5 Jahren korreliert mit Fernsehnutzungszeiten im Alter von 6 Jahren.

117 Fernsehnutzungszeiten im Alter von 6 Jahren korreliert mit Fernsehnutzungszeiten im Alter von unter 3 Jahren. 
sollten und wie der Erfolg einer Maßnahme definiert und gemessen werden kann.

\subsection{Medienbezogenes Risikoverhalten mindern: Zwei theoretische Perspektiven und ihre praktische Umsetzung}

Die öffentliche Debatte über kindliche und jugendliche Mediennutzung und deren problematische Dimensionen und Wirkungen ist insbesondere von einem Schlagwort geprägt: „Medienkompetenz“. Die Förderung von Medienkompetenz von Kindern und Jugendlichen hat einen festen Platz in der politischen Agenda auf Ebene der Europäischen Union, des Bundes und der deutschen Bundesländer und ,steht im Zentrum zahlreicher gesellschafts- und bildungspolitischer Bemühungen (Gysbers, 2008, S. 21; ein Überblick über aktuelle Entwicklungen in Deutschland ebenda auf S. 21 - 25). In Kapitel 3 wurde bereits dargelegt, dass grundsätzlich zwischen dem entwicklungspsychologischen Medienkompetenzbegriff und dem pädagogischen Medienkompetenzbegriff zu trennen ist, wobei die entwicklungspsychologischen Grundlagen von Mediennutzung und Medienkompetenz bereits dargestellt wurden (vgl. S. 45 - 56). Der medienpädagogische Medienkompetenzbegriff leitet sich von der pädagogischen Zielperspektive ab, was wichtige Medienkompetenzen sind und wie sie gelehrt und gelernt werden können (vgl. S. 47 in dieser Arbeit), wobei die jeweilige kognitive, emotionale und soziale Entwicklung von Kindern und Jugendlichen - zumindest implizit - in allen medienpädagogischen Modellen als Grenze medienpädagogischer Bemühungen wohl „,mitgedacht“, jedoch kaum explizit diskutiert wird ${ }^{118}$.

Neben Theorien und Projekten zur Förderung umfassender Medienkompetenz von Kindern und Jugendlichen entwickelte sich in den letzten zehn Jahren eine durch Medienwirkungsforschung, Gesundheitspsychologie und Pädiatrie geprägte Theorie und Praxis, in der die Prävention problematischer Effekte der Mediennutzung im Mittelpunkt stehen. Vor diesem Hintergrund ist das Thema problematischer Mediennutzungsmuster in den letzten Jahren auch Gegenstand der Gesundheitsförderungsforschung beziehungsweise der Public-HealthForschung geworden.

Nachfolgend sollen beide Perspektiven, die vereinfachend MedienkompetenzPerspektive und Health-Promotion-Perspektive benannt sind, kurz dargestellt werden und anschließend daraufhin überprüft werden, welche Elemente dieser

118 Vgl. hierzu auch die Kritik am pädagogischen Medienkompetenzbegriff ab S. 163 in dieser Arbeit. 
Perspektiven fruchtbar bei der Konzeption von Maßnahmen zur Reduktion problematischer Mediennutzungsmuster sein können.

\subsubsection{Die Medienkompetenz-Perspektive}

\subsubsection{Theoretischer Hintergrund}

Mit der Grundlagenarbeit von Baacke (1973) fand der Medienkompetenzbegriff erstmals Eingang in die medienpädagogische Debatte, wobei Baacke zunächst von verschiedenen Dimensionen kommunikativer Kompetenz spricht. Baacke versteht Medienkompetenz als eine systemische Ausdifferenzierung von kommunikativer Kompetenz, indem sich Medienkompetenz in Auseinandersetzung mit den permanenten Veränderungen der Kommunikationsstrukturen durch technisch-industrielle Vorkehrungen und Erweiterungen entwickelt, in denen Menschen sich kommunikativ-handelnd auch mit Medien ausdrücken (Hugger, 2008). Wie Gysbers zutreffend anmerkt, fehlt es dem Medienkompetenzkonzept von Baacke und darauf aufbauenden Ausarbeitungen (etwa Aufenanger, 1997; Schorb, 1997; Tulodziecki, 1998) an einer kompakten Begriffsdefinition (Gysbers, 2008, S. 32), wobei es keinesfalls an Definitionsversuchen mangelt - Gapski listet allein 104 verschiedene Definitionen auf (2001, S. 255 - 293) - sondern an der Kompaktheit. Vielmehr wird das Medienkompetenzverständnis durch Ausdifferenzierung verschiedener Dimensionen von Medienkompetenz näher bestimmt und erläutert (ebenda).

Baacke gliedert Medienkompetenz in die vier Bereiche Medienkritik, Medienkunde, Mediennutzung und Mediengestaltung. Medienkritik bedeutet im Kontext von Baackes Dimensionalisierung die Fähigkeit, problematische gesellschaftliche Prozesse - wie etwa die wirtschaftliche Konzentration von Medienunternehmen - analytisch zu erfassen und dieses Wissen auf sich selbst und das eigene Handeln in ethischer Weise anzuwenden und zu reflektieren. Medienkunde beschreibt das Wissen über das gegenwärtige Mediensystem, welches gegliedert werden kann in eine informative Dimension (klassische Wissensbestände, zum Beispiel über unterschiedliche Filmgenres) und in eine instrumentellqualifikatorische Dimension (etwa die Fähigkeit zur Bedienung einer digitalen Benutzeroberfläche). Kompetenz im Bereich der Mediennutzung gliedert sich in die (jenseits der rein technischen Kompetenz liegende) Kompetenz zur Rezeption medialer Inhalte sowie die Kompetenz zur (interaktiven) Teilhabe an medialer Kommunikation. Mediengestalterische Kompetenz umfasst nach 
Baacke alle Fähigkeiten zur innovativen Veränderung des Mediensystems wie auch zur kreativ-ästhetischen Gestaltung medialer Inhalte.

Baackes Medienkompetenz-Konzept wurde im Laufe der letzten drei Jahrzehnte durch zahlreiche Autoren weiterentwickelt, expliziert und auch kritisiert $^{119}$. So bemerkt Groeben (2004), der normative Gehalt in Baackes Konzept, in dem Medienkompetenz als erstrebenswerte Lernaufgabe definiert wird, erschwere eine Überführung des Konzeptes in ein empirisch zu fassendes Konstrukt (S. 28 - 29) und lasse zudem eine (auf das Individuum anwendbare) psychologische Sichtweise vermissen, durch die Medienkompetenz sich als eine Reihe sich erst entwickelnder Fähigkeiten zeigt, die sich im Wechselspiel mit verschiedenen Sozialisationsinstanzen auch unterschiedlich ausprägen können (ebenda). Auch Hurrelmann kritisiert eine "unbefangene" Übernahme des Kompetenzbegriffs durch die Medienpädagogik aus anderen Wissenschaftsbereichen, die mit dem Bereich der Medien eigentlich nichts zu tun haben (2002, S. 308). Tatsächlich lesen sich Baackes Teildimensionen von Medienkompetenz (Medienkunde, Mediengestaltung, etc.) eher wie ein Lehrplan, als dass sie individuelle Kompetenzen beschreiben.

Obwohl sich Baacke in der Beschreibung seiner Teildimensionen von Medienkompetenz nicht explizit an entwicklungspsychologischen Konzepten wie Weinerts Kompetenzbegriff (vgl. S. 46 in dieser Arbeit) orientiert, ist doch zu berücksichtigen, dass seine Konzeption auch auf entwicklungspsychologische und kognitionspsychologische Konzepte bezogen ist (Baacke, 1999, S. 32 - 34) und dass er im Endeffekt immer auf die individuell notwendigen Fähigkeiten zurückkommt, die zur Erreichung der einzelnen Kompetenzdimensionen gefragt sind. Auch Autoren aktueller Medienkompetenzmodelle bemühen sich, den Begriff der Medienkompetenz an psychologische Konzepte weiter anzunähern. So unterteilt Aufenanger Medienkompetenz in die folgenden Unterdimensionen: die Kognitive, die Moralische, die Soziale, die Affektive, die Ästhetische Dimension sowie die Handlungsdimension (Aufenanger, 1997). Kübler unterscheidet die Unterdimensionen Kognitive Fähigkeiten, Analytische und Evaluative Fähigkeiten, Sozial Reflexive Fähigkeiten sowie Handlungsorientierte Fähigkeiten (Kübler, H.-D., 1999). Schorb differenziert bei Medienkompetenz zwischen Orientierungs- und Strukturwissen, Kritischer Reflexivität, Fähigkeit und Fertigkeit des Medienhandelns und Sozialer, Kreativer Interaktion (Schorb, 1997). Six und Gimmler schließlich unterscheiden zwischen (1) Medienwissen und

119 Eine kompakte Darstellung der wichtigsten Autoren und Konzepte findet sich bei Gysbers (2008, S. 29 - 39), eine umfassende Darstellung des Medienkompetenzbegriffs, seiner Definitionen und Dimensionen bei Gapski (2001). 
Technikkompetenz, (2) Reflexions- und Bewertungskompetenzen, (3) Nutzungsund Verarbeitungskompetenzen sowie (4) Speziellen Kommunikatorkompetenzen (2007b, S. 282 - 285).

Auch Groeben (2004) legt eine eigene Konzeptionalisierung von Medienkompetenz vor, wobei er konsequent Aspekte wie etwa die Medienkritik durch Handlungs- und Erlebensaspekte technischer, kognitiver, emotionalmotivationaler und sozialer Art zu ersetzen sucht (S. 32) und andererseits zu bedenken gibt, dass Medienkompetenz in ein vertikales Raster von Kompetenzen eingeordnet werden können muss (ebenda). Dabei müsse, so Groeben, eine Trennung von übergeordneten Kompetenz-Ebenen gelingen, so dass Medienkompetenz selbst horizontal strukturiert werden kann (S. 33). Eine solche Strukturierung, schlägt Groeben vor, könnte dann nicht hierarchisch sein, sondern (mediennutzungs-)prozessorientiert, wie es etwa Hobbs für das LiteracyKonzept umgesetzt habe (access, analyse, evaluate, and communicate) (ebenda). Damit unternimmt Groeben den Versuch, Medienkompetenzkonzepte der medienpädagogischen Wissenschaft in den (entwicklungs-) psychologischen Kompetenzbegriff $\mathrm{zu}$ integrieren. Vor dem Hintergrund seiner eigenen kommunikationswissenschaftlichen Sichtweise schlägt Groeben eine Strukturierung von Medienkompetenz nach medialen Verarbeitungsprozessen auf verschiedenen qualitativen Ebenen (Kognition, Emotion/Motivation, technikbezogene Handlungskomponente, soziale Handlungskomponente etc.) vor (S. 34 - 40):

- Medienwissen/Medialitätsbewusstsein

- Medienspezifische Rezeptionsmuster

- Medienbezogene Genussfähigkeit

- Medienbezogene Kritikfähigkeit

- Selektion/Kombination von Mediennutzung

- (Produktive) Partizipationsmuster

- Anschlusskommunikation

Groebens Medienkompetenzansatz ist durch die Integration psychologischer und pädagogischer Kompetenzkonzepte und die kommunikationswissenschaftlich begründete prozesshafte Strukturierung von Medienkompetenz als der elaborierteste Versuch anzusehen, Medienkompetenz in ihren Teilaspekten und in Abgrenzung von anderen Kompetenzbereichen $\mathrm{zu}$ beschreiben. Aufgrund dieses Anspruchs und dem aus seiner Sicht starken normativen Gehalt des medienpädagogischen Medienkompetenzbegriffs bleibt Groebens Medienkompetenzkonzept (bewusst) sehr abstrakt und wenig handlungsleitend für die 
konkrete medienpädagogische Arbeit mit Kindern und Jugendlichen. Zwar bieten die unterschiedlichen Teilkompetenzen in Groebens Konzeption viele Ansatzpunkte für die pädagogische Überlegungen zur Stärkung dieser Kompetenzfelder, eine weitergehende Ausarbeitung von Groebens Konzept hin zu einer Leitlinie medienpädagogischen Arbeitens fehlt indes noch vollkommen. Insofern ist es wenig verwunderlich, dass Medienkompetenzkonzepte, die Medienkompetenz nicht in individuell (messbare) Teilkompetenzen im Umgang mit Medien strukturieren, sondern als Ziele konkreter medienerzieherischer Arbeit, deutlich stärker von der pädagogischen Praxis und Politik rezipiert wurden. Das bekannteste Konzept dieser Art stammt von Tulodziecki (1998) und ist inzwischen als Koordinierungsrahmen medienerzieherischer Unterrichtsarbeit von den Bundesländern Nordrhein-Westfalen wie auch Niedersachsen übernommen worden (Gysbers, 2008, S. 34).

Tulodziecki definiert fünf Komponenten von Medienkompetenz: Auswählen und Nutzung von Medienangeboten, eigenes Gestalten von Medienbeiträgen, Verstehen und Bewerten von Mediengestaltungen, Erkennen und Aufarbeiten von Medieneinflüssen, sowie das Durchschauen und Beurteilen von (Medienproduktions- und Verbreitungs-) Bedingungen (1998). In der Explikation der unterschiedlichen Komponenten definiert Tulodziecki konkrete Lernziele für die Arbeit mit Kindern und Jugendlichen und entwirft insofern ein medienpädagogisches Curriculum für schulische und außerschulische Medienerziehungsprogramme.

Auswählen und Nutzen von Medienangeboten bedeutet nach Tulodziecki in der pädagogischen Arbeit, dass Kinder und Jugendliche lernen sollen, das Informationspotential der Medien in angemessener Form zu nutzen. Als besonders vielversprechend erscheint es dem Autor, Kindern und Jugendlichen die Möglichkeit zu geben, Nutzungen zu erproben, Nutzungspläne zu entwickeln und $\mathrm{zu}$ bewerten sowie Handlungsalternativen $\mathrm{zu}$ erfahren und $\mathrm{zu}$ diskutieren (Tulodziecki, 1998, S. 702). Eigenes Gestalten und Verbreiten von Medienbeiträgen wird nach Tulodziecki durch eigene Mediengestaltungsprojekte der Kinder und Jugendlichen erlernt. Konkret schlägt er die Erarbeitung von Dokumentationen, zum Beispiel Fotodokumentationen vor, die exemplarische Verwirklichung publizistischer Produkte wie zum Beispiel Hörmagazine, sowie die eigene Gestaltung fiktionaler und experimenteller Darstellungen etwa in Form von Videofilmproduktionen (Tulodziecki, 1998, S. 702 - 703). Zur Stärkung der Kompetenz zum Verstehen und Bewerten von Mediengestaltungen fordert Tulodziecki die Durchführung von Projekten oder Unterrichtseinheiten, durch welche Kinder und Jugendliche angeregt werden, verschiedene mediale Gestaltungsmittel zu vergleichen und hinsichtlich ihrer besonderen Möglich- 
keiten und Grenzen zu beurteilen (Tulodziecki, 1998, S. 703). Die Erkennung und Aufarbeitung von Medieneinflüssen vermittelt sich gemäß Tulodziecki durch Projekte und Unterrichtseinheiten, die Kindern und Jugendlichen die Existenz von Medienwirkungen in deren Alltagsbereich aufzeigen, zum Beispiel im Gefühlsleben (Spaß, Spannung oder auch Angst), im Bereich der Vorstellungen über alltäglich erfahrbare Gesellschaftsbereiche (zum Beispiel Familie, Arbeit und Politik) oder auch im Bereich der Verhaltensorientierungen, (zum Beispiel im Konflikt- und Freizeitverhalten der Kinder- und Jugendlichen). Auch schlägt der Autor vor, langfristige Medieneinflüsse etwa auf Wertorientierungen oder gesellschaftliche Zusammenhänge aufzuzeigen (Tulodziecki, 1998, S. 703 704). Der letzte Punkt in Tulodzieckis Konzeption von Medienkompetenz ist das Durchschauen und Beurteilen von Bedingungen der Medienproduktion und distribution. Hier soll Kindern und Jugendlichen ermöglicht werden, personale, ökonomische, rechtliche und politische Bedingungen von Medienproduktion und Medienverbreitung in unserer Gesellschaft $\mathrm{zu}$ durchschauen und eigene Einflussmöglichkeiten zu erkennen und zu nutzen. (Tulodziecki, 1998, S. 704).

\subsubsection{Kritik}

Ein Hauptproblem aller pädagogischen Konzepte, die sich auf Medienkompetenz, ihre Vermittlung und Förderung berufen, ist die große Unschärfe des Begriffs. Durch die Breite seines Geltungsanspruches, durch die Vielzahl und ständige Ausdifferenzierung als wichtig erachteter Teildimensionen verliert das Konzept der Medienkompetenz zunehmend an Kontur, empirischer Messbarkeit und letztlich auch - von seiner rein formelhaften Verwendung einmal abgesehen - an Bedeutung für die konkret-medienpädagogische Praxis. Wie oben bereits angeklungen, wird in den Medienkompetenzkonzepten von Baacke, Groeben und Tulodziecki ein zentraler Aspekt von Medienkompetenz permanent gedanklich "mitgeschleift", ohne dass seine Bedeutung für das Verständnis und die Entwicklung von Medienkompetenz explizit und eingehend diskutiert wird: Die Abhängigkeit von Medienkompetenz und den Möglichkeiten ihrer Förderung im schulischen und außerschulischen Kontext mit dem jeweiligen Entwicklungsstand von Kindern und Jugendlichen (vgl. auch Kapitel 3.1). Dies zeigt sich sowohl in Diskussion zu Möglichkeiten und Methoden der Medienkompetenzvermittlung als auch in der Interpretation mediennutzungsbezogener Problemfelder. 


\subsection{Möglichkeiten der Medienkompetenzvermittlung}

Medienkompetenz ist - wie alle Kompetenzen - primär eine Funktion kognitiver Fähigkeiten und Fertigkeiten (vgl. S. 46 in dieser Arbeit). Konzepte zur Steigerung von Medienkompetenz setzen vor diesem Hintergrund auf die Vermittlung von Wissen und medienbezogenen Problemlösungsstrategien. So resümiert Blömeke zur Frage, was Kennzeichen eines guten Medienprojekts im Unterricht aus lehr-lerntheoretischer Sicht sei:

„Gemäßigt konstruktivistische Ansätze bieten - gestützt durch zahlreiche empirische Untersuchungen - den derzeit angemessensten Rahmen, um den Prozess des [medienkompetenzbezogenen] Wissenserwerbs zu erklären. [...] Dies beinhaltet, dass neue Inhalte nicht als fertiges System bzw. als Welt abgeschlossener Erkenntnisse präsentiert werden, sondern dass Lernende zusammen mit anderen Lernenden eigene Wege suchen." Blömeke (2002, S. 9 - 10)

Medienkompetenz ist mithin das Ergebnis eines durch jedes Kind, jeden Jugendlichen individuell unterschiedlich vollzogenen und von außen nur schwer beeinflussbaren Prozesses der Aneignung einer Wissensbasis, die Grundlage medienkompetenten Handelns ist. Als kritisch zu sehen ist nun, dass die Medienkompetenz-Perspektive die Tatsache ausklammert, dass (Medien-) Verhalten gerade von Kindern keineswegs ausschließlich das Ergebnis eines bewussten, wissensbasierten Lernprozesses ist, sondern dass durch Lernprozesse wie dem Modelllernen (vgl. S. 82 in dieser Arbeit) oder Konditionierungsprozesse (Steiner, 2006, S. 139 - 163) gerade jüngere Kinder entscheidend geprägt werden. Medienhandeln kann also auf der Grundlage von Medienkompetenz geschehen, ist aber maßgeblich auch von anderen Lernprozessen gesteuert. Verfechter der Medienkompetenz-Förderung gehen nun davon aus, dass objektiv nicht-funktionales, nicht auf Medienkompetenz gegründetes Medienhandeln durch die Förderung von Medienkompetenz korrigiert werden kann. Dabei klammern sie Ansätze des Verhaltenslernens aus Überlegungen zur Vermittlung angemessener Strategien zum Umgang mit Medien weitgehend aus ${ }^{120}$. So zeigt

120 Wenn hier die Fokussierung der Medienkompetenzforschung auf explizit wissensgesteuerte Lernprozesse und ihre Bedeutung für das Handeln kritisiert wird, bedeutet das nicht, dass im Rahmen sozialer Lernprozesse oder der operanten Konditionierung kognitive Kontrolle ausgeschaltet ist bzw. Wissen über einen Gegenstand keine Rolle spielt für die Handlung. Es soll lediglich deutlich werden, dass Handeln keineswegs immer äquivalent zu vorhandenen Wissensbeständen oder äquivalent zu bestimmten Einstellungen ist, mithin keineswegs zwangläufiges Produkt vorhandener Kompetenzen ist. Insofern ist es ein Fehlschluss zu glauben, dysfunktionales Handeln könne nur durch Wissensvermittlung oder Einstellungsveränderungen geändert werden. Denken beeinflusst zwar immer das Handeln, aber Handeln beeinflusst auch immer das Denken (Gage 
sich die Fokussierung auf wissensbasierte, selbstgesteuerte Lernprozesse auch bei Spanhel:

„Der Aufbau von Medienkompetenz beim Kind ist ein selbstgesteuerter Konstruktionsprozess und kann daher nicht von außen, durch die Eltern oder Erzieher im einzelnen gesteuert oder bestimmt, sondern nur ausgelöst werden. Gezielte medienpädagogische Maßnahmen, wie z. B. Gängelungen, Verbote oder Strafen in der Familie oder direkt auf Veränderung des Medienhandelns gerichtete Konzepte in Kindergarten oder Grundschule werden daher kaum zu Erfolgen führen." (Spanhel, 1999, S. 13)

Wenn Spanhel auch Recht zu geben ist, dass medienpädagogische Maßnahmen wie „Gängelungen, Verbote oder Strafen“ keinesfalls erfolgversprechende Instrumente der Medienkompetenzvermittlung sind, stellt er mit dem letzten Teil seiner Aussage recht pauschal den Sinn schulischer Beeinflussung von Medienverhalten in Frage. Für Spanhel ist funktionales Medienhandeln zwangsläufig Ergebnis von Medienkompetenz. Die Erlangung von Medienkompetenz ist von außen (zum Beispiel Schule) nicht im Einzelnen steuerbar. Dementsprechend vage bleibt seine anschließende Folgerung, was denn Ziel und Inhalte institutioneller Medienkompetenzvermittlung für Kinder sein können:

„Aufgrund der unterschiedlichen sozialen Handlungskontexte ist nicht zu erwarten, dass die in den Erziehungsinstitutionen Kindergarten und Grundschule vermittelten Medienkompetenzen das Medienhandeln der Kinder in den ganz anders strukturierten Handlungsrahmen von Familie und Freizeit nachhaltig verändern. Das Ziel und der pädagogische Gewinn dieser Maßnahmen liegt im Aufbau und in der Einübung möglichst vielfältiger Handlungsalternativen und Problemlösungsmuster mit Medien in unterschiedlichen sozialen Situationen." (Spanhel, 1999, S. 13)

Medienkompetenzvermittlung ist demnach kein geeignetes Instrument der Medienerziehung, wenn es um die Vermittlung kindgerechten, alltäglichen Medienhandelns geht.

\subsection{Medienwirkungen aus Sicht der Medienkompetenz-Perspektive}

Der „Blinde Fleck“ der Medienkompetenzforschung hinsichtlich der Verschiedenartigkeit möglicher Lernprozesse, die beim Umgang mit Medien eine Rolle spielen können, zeigt sich nicht nur im Primat wissensbezogener Unterrichtsgestaltung, sondern auch bei der kritischen Interpretation der Erkenntnisse

\& Berliner, 1996, S. 269). Somit sind auch Handlungsinstruktionen von außen geeignet, Kognitionen, Wissen und Einstellungen zu verändern, sofern die Handlungsweise vom Lernenden im Nachhinein als positiv und zu anderen Wissens- und Einstellungsbeständen passend erachtet wird. 
der Medienwirkungsforschung. So argumentiert Vollbrecht als einer der derzeit prominentesten Vertreter der medienpädagogischen Medienkompetenzforschung gegen die Medienwirkungsforschung:

„Medien wirken nicht durch ihre Inhalte, sondern durch die Passung ihrer Bedeutungsangebote für die jeweiligen Mediennutzer [...] Entscheidend ist also nicht, ob beispielsweise Bilder zur Identifikation und Nachahmung angeboten werden, sondern ob und wie diese Bilder zu unserer psychischen Realität passen [...] und dann entsprechend dieser Realität gedeutet werden.“ (Vollbrecht, 2008, S. 151)

Mit dieser Argumentation greift Vollbrecht zwar einerseits die Erkenntnisse der Medienwirkungsforschung auf, dass vergleichsweise starke (positive wie negative) Medienwirkungen unter anderem dann $\mathrm{zu}$ erwarten sind, wenn eine hohe Passung zwischen medial präsentiertem Setting der Handlung mit dem Alltagsumfeld des (minderjährigen) Rezipienten besteht und wenn die gezeigten Inhalte geeignet sind, bereits vorhandene Wahrnehmungs- und Handlungsschemata zu verstärken (vgl. S. 82 - 107 in dieser Arbeit). Andererseits aber spricht er problematischen Medieninhalten dann jegliche Wirkung ab, wenn die rezipierten Inhalte nicht der psychischen Realität des Rezipienten entsprechen.

Besonders im Bezug auf kindliche Medienrezipienten erscheint eine solche Sichtweise allerdings unzulässig, sind bei dieser Gruppe doch Wahrnehmungsund Handlungsschemata keineswegs gefestigt, und ist hier auch die Unterscheidungsfähigkeit zwischen eigener Realität, rezipierter beziehungsweise gespielter Realität und einem Vergleich beider Sphären keineswegs so stabil, wie Vollbrechts Argumentation suggeriert (vgl. S. 45-56 in dieser Arbeit). Vollkommen zu Recht weist Spanhel (2002, S. 2) in diesem Zusammenhang darauf hin, dass Kinder heute von Geburt an in hohem Maße medialen Eindrücken und Einflüssen, symbolischen Bedeutungen und Beziehungsbotschaften ausgesetzt seien, die nicht für sie gemacht und bestimmt seien, und die sie nicht (in adäquater Weise) verstehen und bearbeiten können (ebenda). Spanhel betont, dass aufgrund mangelnder empirischer Forschung gerade im Bereich der frühkindlichen Medienwirkungsforschung gar nicht genau abzusehen sei, welche nicht kontrollierbaren, den Entwicklungsprozess beeinträchtigenden oder förderliche Wirkungen Medien haben könnten. Bereits in dieser frühen Entwicklungsphase, so der Autor, bildeten sich später nur schwer korrigierbare Rezeptionsund Handlungsroutinen aus (ebenda). Dies entspricht auch den oben angeführten empirischen Erkenntnissen zur relativen Stabilität individueller Mediennutzungsmuster (vgl. S. 156 - 157 in dieser Arbeit). Bereits kleine Kinder entwickeln, so Spanhel an anderer Stelle, ein System von Wahrnehmungs-, Rezeptions-, Gefühls-, Wertungs- und Handlungsmustern, die sich durch vielfältige Wiederholungen in spezifischen Formen stabilisieren (Spanhel, 1999, 
S. 10). Eine solche, auf individuellen Erfahrungen gegründete Medienkompetenz sei deshalb problematisch, weil eben diese Erfahrungen einseitig, auf problematische Inhalte ausgerichtet, und mit entwicklungsgefährdenden Einflüssen verbunden sein könnten (Spanhel, 2002, S. 4). Ergänzen lassen sich diese Überlegungen um die Erkenntnis Tulodzieckis, dass Medienkompetenz, hier verstanden als die Fähigkeit zur Nutzung altersgerechter Medieninhalte in einem zeitlich unproblematischen Rahmen, untrennbar mit der intellektuellen und sozial-moralischen Entwicklung von Kindern und Jugendlichen verbunden ist, die (auch) von familiären und anderen sozialen Rahmenbedingungen mitgeprägt wird (Tulodziecki, 1988).

Insofern erscheinen auch die (teilweise gut begründeten) Vorbehalte der Medienkompetenzforschung vor bewahrpädagogischen Ansätzen dann als unzulässig, wenn, wie in Hoffmanns Darstellung bewahrpädagogischer Ansätze im Handbuch Medienpädagogik, in wenig differenzierter Weise Bemühungen kritisiert werden, Kinder und Jugendliche vor explizit problematischen Medieninhalten zu schützen:

„Die Grundannahmen der Bewahrpädagogen sprechen den Kindern und Heranwachsenden die Fähigkeit ab, eine medienbezogene Selbstständigkeit zu zeigen und weiter zu entwickeln. Es besteht zwar weitgehend Konsens, dass Kinder und Jugendliche bis zu einem gewissen Grade schutzbedürftig sind; dennoch muss es Ziel jeder Erziehung bleiben, Eigenverantwortlichkeit und Selbstbestimmung bzw. Mündigkeit zu erreichen und immer schon anzunehmen. Die Erkenntnisse der Medienforschung über das komplexe Wirkungsgefüge bei der Mediennutzung bleiben bei vorrangig bewahrenden Ansätzen weitgehend unbeachtet.“ (Hoffmann, 2008, S. 49)

Frappierend unausgegoren erscheint an dieser Argumentation die Gleichstellung (vernünftiger) medienerzieherischer Ziele mit der Feststellung, Selbstbestimmung beziehungsweise Mündigkeit sei „immer schon anzunehmen“. Die Aussage, es bestehe „zwar weitgehend Konsens, dass Kinder und Jugendliche bis zu einem gewissen Grade schutzbedürftig sind“, wirkt vor diesem Hintergrund als inhaltsleer, kann doch bei jeder Bemühung, Kinder vor problematischen Medieninhalten zu schützen, kritisiert werden, die Maßnahme missachte die Selbstbestimmung der Betroffenen und verhindere die Entwicklung medienbezogener Selbständigkeit.

\subsubsection{Förderung von Medienkompetenz - Empirische Forschungslage}

Bei der Sichtung der Literatur zur Medienkompetenzförderung erscheint auffällig, wie wenig die theoretischen Überlegungen zur Medienkompetenz bisher mithilfe empirischer Studien überprüft wurden. Grundsätzlich lässt sich 
zwischen Studien unterscheiden, die die Notwendigkeit medienpädagogischer Aktivitäten im Hinblick auf vorher definierte Zielparameter empirisch überprüfen, Studien, die allgemein die Verbreitung von Aktivitäten zur Medienkompetenzförderung beziehungsweise Medienerziehung erheben, und solchen Studien, die gezielt die Effektivität solcher Maßnahmen messen. Die Notwendigkeit medienerzieherischer Maßnahmen wurde im Rahmen dieser Arbeit bereits belegt (vgl. S. 125 - 130 in dieser Arbeit). Insofern soll an dieser Stelle dargestellt werden, inwieweit die Verbreitung von Programmen zur Förderung von Medienkompetenz in Deutschland empirisch untersucht wurde und inwiefern es bisher gelungen ist, konkrete Medienkompetenzprojekte empirisch zu evaluieren. Dabei sollen solche Projekte und Programme beleuchtet werden, die eine eindeutig medienpädagogische Prägung haben und aus Überlegungen zur Stärkung von Medienkompetenz hervorgegangen sind.

\subsection{Status Quo institutioneller Medienerziehung für Kinder}

Repräsentative Studien zur allgemeinen Verbreitung von Programen zur Förderung von Medienkompetenz beziehungsweise zu Medienerziehungsinhalten in Deutschland liegen derzeit für nordrhein-westfälische Grundschulen (Tulodziecki et al., 2000) und Kindergärten (Six \& Gimmler, 2007a) vor, sowie für niedersächsische Kindergärten (Schneider, Scherer, Gonser \& Tiele, 2010) und Schulen (Gysbers, 2008). Grundsätzlich zeigte sich dabei vom Kindergarten bis zum Sekundarschulbereich, dass Medienerziehung in Kindergarten und Vorschule eine eher geringe Rolle im pädagogischen Alltag spielt. Und dies, obwohl sowohl unter Erzieherinnen und Erziehern in Kindergärten als auch unter Lehrkräften eine Sensibilität für problematische Mediennutzung von Kindern und Jugendlichen eher die Regel ist, und obwohl beide Berufsgruppen durchaus der Meinung sind, dass kindliche Alltagsmediennutzung und mögliche problematische Folgen durchaus im Rahmen der pädagogischen Arbeit in Kindergarten und Schule bearbeitet werden sollten (Gysbers, 2008; Schneider et al., 2010; Six \& Gimmler, 2007a; Tulodziecki et al., 2000).

Six und Gimmler (2007a) resümieren für den Kindergartenbereich, dass medienpädagogische Handlungsformen im Jahr 2006, „nach wie vor recht selten" waren und nicht häufiger stattfanden als im Jahr 1997, aus dem eine vergleichbare Untersuchung der gleichen Autorengruppe vorliegt (Six, Frey \& Gimmler, 1998). Auf einem siebenstufigen Index für praktische Medienerziehung im Kindergarten, den Six und Gimmler (2007a) im Rahmen ihrer Studie berechneten (Einzelheiten zur Indexbildung bei Six \& Gimmler, 2007a, S. 243), 
erhielten 29,5 Prozent aller befragten Erzieherinnen und Erzieher 0 bis 1 Punkte, was die Autoren als ,defizitäre Medienerziehung“ bezeichnen, weitere 26,6 Prozent der Befragten erreichten 2 Punkte (,wenig Medienerziehung“"). Nur 23,3 Prozent praktizierten eine „moderat engagierte Medienerziehung“ (3 Punkte) und 21 Prozent eine „intensive Medienerziehung“ (4-6 Punkte) (S. 244 - 245). Schneider et al. (2010) kommen insgesamt zu dem Schluss, dass die generell sehr skeptischen Befunde von Six und Gimmler (2007a) zum Status Quo medienerzieherischer Aktivitäten in nordrhein-westfälischen Kindergärten durch die eigene niedersächsische Studie ,in vollem Umfang unterstützt werden.“ (Schneider et al., 2010, S. 111). Zudem identifizieren sie zwei Schlüsselfaktoren medienerzieherischen Handelns im Kindergarten: Motivation und Kompetenz (S. 112 -113). So schätzen sich Erzieherinnen und Erzieher selbst als wenig medienkompetent ein und fühlen sich von der Vielfalt aktueller Medienangebote eher überfordert. Dies hängt nach Schneider et al. auch mit Defiziten in der Ausund Weitebildung der Erzieherinnen und Erziehern zusammen. Zudem räumen Erzieherinnen und Erzieher - trotz eines durchaus vorhandenen Problembewusstseins im Hinblick auf die (teilweise) problematische Mediennutzung ihrer Kinder - Medienerziehung einen eher niedrigen Stellenwert unter verschiedenen pädagogischen Zielsetzungen ein (ebenda).

In der Grundschule nimmt die Häufigkeit medienerzieherischer Aktivitäten zwar zu, 49,9 Prozent der im Jahr 2000 befragten Grundschullehrerinnen und lehrer gab jedoch an, im laufenden Schuljahr noch keine Unterrichtseinheit mit diesem Thema durchgeführt zu haben (Six, Frey \& Gimmler, 2000, S. 186). Gysbers (2008, S. 62) weist in diesem Zusammenhang allerdings zu Recht darauf hin, dass zum Befragungszeitpunkt das laufende Schulhalbjahr erst zur Hälfte vorbei war und die Zahlen insofern medienerzieherische Aktivitäten in der Grundschule eher unterschätzen.

Gysbers selbst verzichtete im Rahmen seiner Befragung niedersächsischer Primar- und Sekundarschullehrerinnen und -lehrer auf den konkreten Bezugspunkt „laufendes Schuljahr“ und befragte die Lehrkräfte allgemein, ob sie bestimmte medienerzieherische Projekte bereits im Unterricht durchgeführt haben. Dabei zeigte sich, dass am häufigsten bereits die Beeinflussung durch Medien (zum Beispiel durch Werbung) im Unterricht zum Thema gemacht wurde (74\% der Befragten gab an, bereits ein entsprechendes Projekt durchgeführt zu haben), es folgten die kritische Analyse von Medienangeboten (57\%), die Analyse des Stellenwertes der Medien für die Schülerinnen und Schüler (53\%), die eigene Gestaltung von Medienangeboten (51\%), sowie drei weitere, deutlich weniger berichtete Inhalte (S. 138 - 139). 
Auffällig erscheint, dass Grundschullehrerinnen und -lehrer in Gysbers Befragung durchweg deutlich weniger von medienerzieherischen Aktivitäten berichten als Sekundarstufenlehrkräfte. So berichteten beispielsweise 44 Prozent der Grundschullehrkräfte, noch nie die Beeinflussung durch Medien (zum Beispiel durch Werbung) zum Thema gemacht zu haben, während dies unter Lehrkräften der Sekundarstufe lediglich 16 (Hauptschullehrerinnen und -lehrer) bis 26 Prozent (Gymnasiallehrerinnen und -lehrer) angaben (S. 247). Ebenso gaben 63 Prozent der Lehrkräfte an Grundschulen an, noch keine kritischen Analysen von Medienangeboten durchgeführt zu haben, während dies in der Sekundarstufe deutlich häufiger geschah (Unterer Wert bei Gesamtschullehrerinnen und lehrern mit $27 \%$, oberer Wert bei Realschullehrkräften mit $41 \%$ ) (ebenda).

Der Einfluss des Elternhauses auf Medienkompetenz und Mediennutzung ihrer Kinder wird von Seiten der Medienpädagogik immer wieder betont (vgl. etwa Spanhel, 1999) und konnte empirisch eindeutig nachgewiesen werden (vgl. S. 125 - 130 in dieser Arbeit). Insofern ist es nicht verwunderlich, dass viele Lehrerinnen und Lehrer das Elternhaus bei Medienkompetenzförderung und Medienerziehung am stärksten in der Pflicht sehen (vgl. bspw. Gallasch, 2000, S. 261 - 265; Gysbers, 2008, S. 153) und Elternarbeit zur Medienerziehung von Kindern bei Erzieherinnen und Erziehern im Kindergarten als noch zentraler angesehen wird als die Medienerziehung der Kinder (Six \& Gimmler, 2007a, S. 207). Dennoch gaben rund ein Drittel der Kindergartenerzieherinnen und erzieher (S. 260) und rund ein Viertel der Grundschullehrkräfte (Six et al., 2000, S. 186) an, selten oder nie Elterngespräche oder Elternabende zu diesem Thema durchzuführen. Die Kindergartenstudie von Six und Gimmler (2007a, S. 260) zeigt, dass solche Elterngespräche, wenn sie denn geführt werden, in der Regel kurze, anlassbezogene Einzelgespräche sind, während Elternabende zu diesem Thema mit 14,1 Prozent eher die Ausnahme sind.

Insgesamt lässt sich festhalten, dass eine institutionell verankerte Medienerziehung in Kindergarten und Schule noch eher die Ausnahme als die Regel ist und dass der Dialog zwischen Eltern, Erzieherinnen und Erziehern sowie Lehrkräften über die Mediennutzung der Kinder noch in unzureichendem Maß Platz im pädagogischen Alltag gefunden hat. Die Autoren der verschiedenen empirischen Studien zu Medienkompetenzförderung und Medienerziehung in Kindergärten und Schulen haben eine ganze Reihe von Faktoren identifiziert, die für den eher unbefriedigenden Status Quo verantwortlich sind. Insbesondere werden fehlenden eigene Medienkompetenzen und - damit zusammenhängend geringe medienerzieherische Motivation genannt.

Vor diesem Hintergrund werden von allen genannten Autoren dringende Verbesserungen bei Umfang, Qualität, Praxisnähe und Verbindlichkeit von 
pädagogischer Aus- und Weiterbildung bezüglich medienerzieherischer Inhalte angemahnt (Gysbers, 2008, S. 17 - 20; Schneider et al., 2010, S. 113; Six \& Gimmler, 2007a, S. 291 - 296). Beziehen sich viele dieser Forderungen auf die Ebene der institutionellen Ausbildung pädagogischer Fachkräfte in Kindergärten und Schulen sowie auf Grundsatzentscheidungen der Bildungspolitik, soll im Folgenden ausschließlich auf diejenigen Punkte eingegangen werden, die im Rahmen einer eigenen Maßnahme zur Prävention medienbezogenen Risikoverhaltens von Minderjährigen zu beeinflussen wären. Ausgangspunkt dieser Überlegungen soll dabei das Leitbild eines Pädagogen sein, der Medienerziehung als integralen Teil seiner Arbeit erfolgreich praktiziert. Hier ist insbesondere die Typologie von Gysbers zu beachten, der mit dem Engagierten Medienprofi einen empirisch nachweisbaren Lehrer-Typus gefunden hat, welcher mit 15 Prozent Anteil unter der von Gysbers befragten niedersächsischen Lehrerschaft allerdings die kleinste der von Gysbers identifizierten Typengruppen bildet (Gysbers, 2008, S. 166 - 177). Dieser Lehrertypus sei, so Gysbers, in allen Bereichen schulischer Medienpädagogik überdurchschnittlich aktiv. Dies gelte sowohl für den didaktischen Einsatz von Medien als auch insbesondere für den Bereich der Medienerziehung (S. 15). Den Engagierten Medienprofi zeichnen neben informationstechnischem Grundlagenwissen sein breites medienerzieherisches Grundlagenwissen sowie seine Kenntnis über die Medienwelt der eigenen Schülerinnen und Schüler aus. Der Typus des Engagierten Medienprofis findet sich vor allem in sprachlichen und gesellschaftswissenschaftlichen Fächern, ist in Grundschulen allerdings vergleichsweise wenig zu finden. Frauen sind in dieser Gruppe im Vergleich zu ihrem Anteil an der Gesamtlehrerschaft deutlich unterrepräsentiert. Interessant erscheint, dass dieser Lehrertypus den Medienumgang der eigenen Schülerschaft vergleichweise wenig kritisch sieht. Diese Aussage erscheint jedoch angesichts der von Gysbers eingesetzten Items zur Erfassung dieser Dimension nur eingeschränkt gültig, da mithilfe der von ihm gewählten Items eine unreflektiert-kulturpessimistische Kritik gemessen wird (Gysbers, 2008, S. 152), keine informiert-kritische Haltung, die von einem Experten des Typs Engagierter Medienprofi, der gerade auch medienerzieherisch stark aktiv ist, eher zu erwarten wäre.

Was unterscheidet nun die durchschnittliche Lehrkraft oder Erzieherin von diesem Leitbild? Bezogen auf den Kindergarten zeigt die Befragung von Six und Gimmler (2007a, S. 274) - wie analog die Befragung von Schneider et al. (2010)-, dass Erzieherinnen und Erzieher insbesondere über fehlende medienpädagogische Kompetenz als auch fehlende eigene Medienkompetenz klagen. Auch fehle es ihnen an klaren Einsichten zu theoretischen Grundlagen und praktischen Möglichkeiten medienerzieherischen Handelns. Zudem mangele es 
Erzieherinnen und Erzieherin, so die Autoren, an konkreten Kenntnissen zum zeitlichen Ausmaß und den Inhalten kindlicher Mediennutzung, was häufig zu einer gesteigerten, aber unreflektiert-unspezifischen Problemwahrnehmung führe, vor dem Hintergrund fehlender medienerzieherischer Kompetenzen allerdings ohne Konsequenzen in Form medienerzieherischen Handelns bleibe (2007a, S.168-184). Bezogen auf die mangelnde Thematisierung kindlicher Mediennutzung in Elterngesprächen ist dieser Einschätzung der Autoren sicherlich recht $\mathrm{zu}$ geben. Allerdings erscheint es auch verständlich, dass Erzieherinnen und Erzieher die kindliche Mediennutzung im täglichen Umgang mit den Kindern eher selten aufgreifen. Dass sich die Erzieherinnen und Erzieher in dem Glauben, die durchschnittlichen Medienzeiten lägen deutlich über ${ }^{121}$ einer Grenze, ab der schädliche Einflüsse vermutet werden können ${ }^{122}$, bemühen, im Rahmen ihrer pädagogischen Arbeit einen alternativen, „medienfreien“ Ausgleich zu schaffen, erscheint zunächst einmal plausibel und - zumindest im Kindergartenalter - auch angemessen.

Als viel problematischer als das Handeln von Erzieherinnen und Erziehern im Kindergarten sind die Befunde von Six et al. (2000, S. 139 - 145) anzusehen, nach denen Grundschullehrkräfte ähnlich wenig medienerzieherisches Engagement zeigen wie Kindergärtnerinnen und Kindergärtner und insgesamt als medienskeptisch zu bezeichnen sind, auf der anderen Seite aber auch verhältnismäßig vage Vorstellungen über mögliche negative wie positive Effekte der kindlichen Mediennutzung haben. Als Konsequenz fordert Gysbers deutlich praxisorientiertere Fortbildungsangebote für Lehrkräfte, die stärker konkret medienerzieherische Themen berücksichtigen (2008, S. 18). Zudem weist er darauf hin, dass Lehrkräften praxisorientierte, didaktisch und methodisch fundierte Materialien insbesondere zur Medienerziehung zur Verfügung gestellt werden müssen (S. 19). Und schließlich fordert er, dass für verschiedene Zielgruppen innerhalb der Lehrerschaft, beispielsweise die medienerzieherisch

121 Tatsächlich überschätzen die Erzieher/innen die tägliche zeitliche Mediennutzung von Kindergartenkindern recht deutlich. So gehen sie im Durchschnitt von 127,32 Minuten täglicher Fernsehzeit und 92.07 Minuten täglicher Computerspielzeit aus (Six \& Gimmler, 2007a, S.155-157).

122 Hier wiederum erscheint es doch erstaunlich, dass die Antworten der Erzieher/inne/n auf die von Six und Gimmler gestellte Frage, ab welchen täglichen Mediennutzungszeiten Erzieher/inne/n schädliche Einflüsse auf Kinder befürchten, sehr nahe an den zeitlichen Grenzen liegen, die in Kapitel 6 auf der Grundlage der Erkenntnisse der Medienwirkungsforschung abgeleitet wurden. So nimmt der Durchschnitt aller Befragten an, ab einer Grenze von 47,17 Minuten täglicher Fernsehnutzung bzw. 34,73 Minuten täglicher Computerspielnutzung seien schädliche Effekte der Mediennutzung auf Kinder im Kindergartenalter zu befürchten (2007a, S.171). 
vergleichweise wenig aktive Gruppe der Frauen, spezielle Angebote gemacht werden sollten (ebenda).

\subsection{Evaluierte Maßnahmen zur Förderung von Medienkompetenz}

Konkrete Maßnahmen oder Unterrichtseinheiten zur Stärkung von Medienkompetenz mit eindeutig medienpädagogischer Prägung sind national wie international kaum umfassend dokumentiert. Das mag zunächst verwundern, da es an Materialien und Anregungen zu medienpädagogischer Arbeit im schulischen Rahmen nicht fehlt. So stellt Tulodziecki (2008) fest, dass schon "[...] zahlreiche Materialien und Handreichungen, Unterrichts- und Projektbeispiele zur Medienerziehung in Familie, Kindergarten, Schule, Jugendarbeit, Hochschule und Erwachsenenbildung entstanden sind" (S. 112). Bereits für das Jahr 1995 berichtet er von 470 Unterrichts- und Projektbeispielen zur Medienerziehung und verweist mit Bezug auf den Folgezeitraum auf vielfältige Veröffentlichungen mit Projekt und Unterrichtsbeispielen für verschiedene Zielgruppen (ebenda). Neben Publikationen aus dem Printbereich seien auch im Internet zahlreiche Projektund Unterrichtsbeispiele, Empfehlungen und Hinweise zur Medienerziehung beziehungsweise Medienbildung zu finden (ebenda).

Dennoch ist hier zu unterscheiden zwischen sehr allgemeinen medienerzieherischen Inhalten und spezifischen Programmen mit klar definierten medienerzieherischen Zielen. So definiert Schorb (2005) Medienerziehung als "pädagogisches Handeln, das zur richtigen, d h. kritisch-reflexiven Aneignung der Medien anleiten soll (S. 240), während Tulodziecki unter Medienerziehung "alle Aktivitäten und Überlegungen in Erziehung und Bildung [versteht] [...], die das Ziel haben, ein humanes bzw. verantwortliches Handeln im Zusammenhang mit der Mediennutzung und Mediengestaltung zu entwickeln" (2008, S. 110). Dieses breite Verständnis von Medienerziehung hat in der Vergangenheit mit dazu beigetragen, dass in der ohnehin stark heterogenen, föderalen deutschen Bildungslandschaft eine Vielzahl medienerzieherischer Konzepte und Projekte existiert, deren systematische Erfassung und Beurteilung bisher nicht gelungen ist. So stellt Tulodziecki dann auch fest, dass aufgrund einer unzureichenden Datengrundlage nicht umfassend geklärt sei, in welchem Umfang und mit welcher Qualität Medienerziehung in der Praxis stattfinde (Tulodziecki, 2008, S. 113). Diese Aussage zeigt, dass nicht nur die empirische Erfassung konkreter medienerzieherischer Maßnahmen an deutschen Schulen noch am Anfang steht, sondern dass derzeit vollkommen ungeklärt ist, wie es um die Qualität dieser Aktivitäten bestellt ist. Obwohl im Bildungsbereich seit einigen Jahren - nicht 
zuletzt unter dem Eindruck der lediglich durchschnittlichen Ergebnisse deutscher Schülerinnen und Schüler bei international vergleichenden Bildungsstudien wie PISA- und TIMSS - Qualität, Qualitätsstandards und Evaluation der Qualität von Unterricht einen hohen Stellenwert haben (vgl. etwa Altrichter \& Heinrich, 2006; Böttcher, Holtappels \& Brohm, 2006; Roeder, 2003), liegen Studien zur Effektivität und Qualität medienpädagogischer Angebote in Deutschland kaum vor.

Brauner (2007) verweist darauf, dass im deutschsprachigen Raum neben Ihrer eigenen Arbeit lediglich ein weiteres evaluiertes Medienkompetenzprojekt (Pöttinger, 1997) publiziert sei. Eigene Recherchen kamen - zumindest für den schulischen Bereich - zu dem gleichen Ergebnis. Das von Brauner evaluierte Projekt richtet sich an Schülerinnen und Schüler ab der achten Klasse mit dem Ziel, insbesondere das Fernsehnachrichtenverständnis der Schülerinnen und Schüler zu verbessern und ihnen eine objektive Auseinandersetzung mit (Nachrichten-)Informationen zu ermöglichen (Brauner, 2007, S. 88). Die Unterrichtseinheit war als außerhalb der Schule stattfindender Projektunterricht konzipiert, in dessen Rahmen an insgesamt sieben Schultagen zur Entstehung von Fernsehnachrichten gearbeitet wurde (S. 90 - 91). Nach zwei theoretischen Einführungstagen, an denen Hintergrundwissen über journalistische Strukturen und Arbeitsweisen sowie zu verschiedenen Präsentationsformen und Erzähl- und Bildstrukturen der Fernsehberichterstattung vermittelt wurden, folgte eine fünftägige Praxiswoche, innerhalb derer die Schülerinnen und Schüler das Gelernte durch eigene Erfahrungen vertieften. Dabei produzierten sie eine eigene Fernsehnachrichtensendung, die am Ende der Projektwoche live über einen Offenen Kanal der Region ausgestrahlt wurde (ebenda). Brauner untersuchte im Rahmen eines Kontrollgruppendesigns das Wissen, die Fertigkeiten und die Einstellungen bezüglich der Konstitution von Nachrichtenmedieninhalten, der Wissensbeschaffung und bezüglich der Meinungsbildung von hessischen Schülerinnen und Schülern neunter bis elfter Klassen unterschiedlicher Schulformen. Während einer ersten Unterrichtsphase wurden $\mathrm{N}=141$ Schülerinnen und Schüler untersucht, während einer zweiten Unterrichtsphase $N=147$ Schülerinnen und Schüler. Dabei konnte ein signifikant deutlicher Wissenszuwachs in allen Bereichen der Nachrichtenmedienherstellung festgestellt werden sowie tendenzielle Leistungssteigerungen im Fertigkeitenbereich der Schülerinnen und Schüler (S. 221).

Das von Pöttinger (1997) entwickelte und selbst evaluierte Unterrichtsprojekt zur Verbesserung von Medienkompetenz richtete sich an Schülerinnen und Schüler erster Grundschulklassen und umfasste eine zehn Doppelstunden dauernde Unterrichtseinheit (S. 201 - 230). Ziel des Projektes war es, die 
Medienkompetenz der Teilnehmerinnen und Teilnehmer im Hörspielbereich zu stärken. Konkret sollten die Schülerinnen und Schüler nach Ablauf der Einheit in der Lage sein, Hörspielprodukte angemessen zu erkennen und zu interpretieren (Wahrnehmungskompetenz), sie adäquat in ihrem Alltag auszuwählen, zu nutzen und darüber zu kommunizieren (Nutzungskompetenz) und Hörspiele selbst zu gestalten, zu produzieren und zu veröffentlichen (Handlungskompetenz) (S. 96). Der Unterricht wurde - mit Unterstützung der Klassenleitung - von der Autorin selbst geleitet und im Januar 1995 mit einer ersten Klasse $(\mathrm{N}=24)$ einer Freiburger Grundschule durchgeführt. Ein zu drei Zeitpunkten (Pre, Post und Follow-up nach vier Monaten) ausgeteilter Fragebogen wurde zudem von einer Kontrollklasse $(\mathrm{N}=23)$ derselben Schule ausgefüllt. Mit qualitativen Einzelinterviews mit ausgewählten Schülerinnen und Schülern und einem quantitativen Fragebogen, der sich auch durch Erstklässler bearbeiten ließ, legte die Autorin deskriptive Daten vor, die nach ihrer Einschätzung den Schluss zulassen, dass sich die Wahrnehmungskompetenz der Kinder durch die Hörspielproduktion verbessert hat, während die Nutzungskompetenz der Kinder durch das Hörspielprojekt nicht und die Handlungskompetenz nur langsam und individuell sehr unterschiedlich entwickelte (S. 265 - 266). Empirisch belegen konnte sie diese Folgerungen lediglich bezüglich der Wahrnehmungskompetenz (und auch hier nicht durch inferenzstatistische Tests).

Neben den oben genannten Unterrichtsprojekten sind für Deutschland weitere medienpädagogische Projekte mit systematischer Begleitforschung lediglich für den Vorschulbereich vorhanden. So wurde die Verwendung von Computern beziehungsweise speziellen Computerprogrammen im Rahmen von Kindergartenprojekten durch Studien von Kochan und Schröter (2006) sowie durch Aufenanger und Gerlach (2008) untersucht. Ziel der Studie von Kochan und Schröter war es, die Wirksamkeit des Einsatzes der vom Hersteller Microsoft entwickelten Software „Schlaumäuse - Kinder entdecken Sprache“ hinsichtlich der Verbesserung der Sprachbildung, insbesondere der Anbahnung des Schriftspracherwerbs im Vorschulalter zu überprüfen (Kochan \& Schröter, 2006, S. 10). Die Schlaumäuse-Software ist ein aus sechs Modulen bestehendes Computerprogramm, in dem Kinder spielerisch ihren Wortschatz erweitern und an Schriftsprache herangeführt werden (zu den einzelnen Modulen vgl. S. 80 der Studie). Die Studie wurde in zwei Staffeln mit jeweils 100 Kindergärten von September 2003 bis Juni 2004 beziehungsweise von September 2004 bis Juni 2005 durchgeführt (S. 12). In den teilnehmenden Kindergärten arbeiteten $\mathrm{N}=3.964$ drei- bis siebenjährige Kinder mit der Software (S. 13). Als Datenbasis der Untersuchung dienten die Einschätzungen der $\mathrm{N}=462$ in den Kindergartengruppen beschäftigten Erzieherinnen und Erzieher zum Umgang der 
Kinder mit dem Programm und ihrer sprachlichen Entwicklung während und nach dem Untersuchungszeitraum sowie die Angaben von $\mathrm{N}=2.137$ Eltern, die einen Fragebogen zur allgemeinen und zur sprachlichen Entwicklung ihres Kindes ausfüllten (da der Fragebogen an alle Eltern verteilt wurde, entspricht dies einer Ausschöpfungsquote von knapp 54 Prozent (S. 15).

Hinsichtlich des Projekterfolgs ziehen die Autoren ein geradezu enthusiastisches Fazit, wobei sie sich hauptsächlich auf die Angaben der Erzieherinnen und Erzieher zum Lernerfolg der Kinder stützen. So berichten sie, dass die Kinder nicht nur schnell lernten, den Computer selbstständig zu bedienen und zu nutzen, sondern dass Kinder neben ihrem Sozialverhalten auch ihre (schrift-) sprachlichen Fähigkeiten deutlich verbesserten, dass sie "Schreiben als Ausdrucksform und Lesen als Informationsentnahme erfahren und praktiziert und diese Fähigkeiten auch in ihr Alltagsleben übernommen [hätten], indem sie davon auch zu Hause freiwillig Gebrauch machten" (S. 71). Zudem verweisen die Autoren auf Berichte der Erzieherinnen, "dass sie - gemäß Bielefelder Screening ${ }^{123}$ - in der Schlaumäuse-Gruppe deutlich weniger Risikokinder bzgl. späterer Lese-Rechtschreib-Schwierigkeiten hatten als früher" (ebenda).

Es ergeben sich bei Lektüre der Studie allerdings einige gravierende Kritikpunkte: Zunächst wurde die Frage nach den Verbesserungen der (schrift-) sprachlichen Fähigkeiten der Kinder nur aufgrund der Angaben der Erzieherinnen und Erzieher und aufgrund einiger allgemeiner Elternangaben untersucht. Auch wurden Erzieherinnen beziehungsweise Erzieher sowie die Eltern zu den Verbesserungen der Kinder nur einmal, am Ende des Untersuchungszeitraums, befragt. Vorher- Nachher-Befragungen $\mathrm{zu}$ diesem Punkt oder standardisierte Tests wurden nicht durchgeführt. Die Autoren der Studie bemerken hierzu, dass "Tests, die lediglich momentane Leistungen zum Testzeitpunkt und unter nicht alltäglichen Bedingungen ermitteln, [...] nicht durchgeführt [wurden]" (S. 11) ${ }^{124}$. Da allen Kindern in den Untersuchungsgruppen die Schlaumäuse-Software zur Verfügung stand, ergibt sich zudem das Problem, dass die Resultate des Projektes nicht mit Entwicklungen in einer Kontrollgruppe untersucht werden konnten. Da anzunehmen ist, dass sich die (schrift-) sprachlichen Fähigkeiten von Kindern zwischen drei und sieben Jahren innerhalb eines

123 Bielefelder Screening zur Früherkennung von Lese-Rechtschreibschwierigkeiten (BISC) (Jansen, Mannhaupt, Marx \& Skowrone, 1990).

124 Das ist insofern erstaunlich, als die Autoren keine methodischen Probleme damit haben, die mündlichen Berichte von Erzieher/inne/n über die allgemeinen Resultate eines Tests wie des BISC als einen zentralen Erfolg des Projektes zu dokumentieren. Die erkenntnistheoretischen Implikationen dieser paradoxen Argumentation auszuleuchten, wäre ohne Frage interessant, allerdings nicht im Rahmen einer wissenschaftlichen Arbeit. 
halben Jahres ohnehin verbessern ${ }^{125}$, ergeben sich deutliche Zweifel an der Validität der Ergebnisse.

Die Studie von Aufenanger und Gerlach (2008) untersuchte in den Jahren 2003 und 2004 ein Jahr lang den Einfluss der Computernutzung in sechs hessischen Kindertagesstätten auf computerbezogene Medienkompetenzen und die kognitive Entwicklung von Kindergartenkindern. Je nach Computernutzung und -erfahrung in den untersuchten Kindertagesstätten wurde in sechs Kindergartengruppen unterschiedlicher Einrichtungen eine Computerinfrastruktur aufgebaut oder ergänzt, zusätzlich wurden pädagogische Konzepte zum Computerumgang in den Kindertagesstätten erarbeitet beziehungsweise konkretisiert. Neben Befragungen von Eltern, Erzieherinnen und Erziehern (Einstellungen zur Computernutzung durch Kinder; Umgang des Computers im kindlichen Alltag) wurden zum Anfang und zum Ende des Projektes die Computerkompetenz sowie kognitive Leistungsfähigkeit der Kinder gemessen. Die Computerkompetenz wurde durch zwei Aufgabenblöcke gemessen, wobei die Kinder innerhalb des ersten Blockes bestimmte Aufgaben am Computer zu erledigen hatten und in einem zweiten Block ein bisher unbekanntes Edutainment-Programm erkunden mussten. Kognitive Leistungsfähigkeit wurde mithilfe der Kaufman Assessment Battery for Children (K-ABC) gemessen (Melchers \& Preuß, 2003). Untersucht wurden 132 Kinder im Alter von durchschnittlich 4,8 Jahren in sechs verschiedenen Einrichtungen, wobei 131 Kinder an der Pre-Messung und 109 Kinder an der Post-Messung teilnahmen. 110 Kinder besuchten eine der sechs Gruppen, in denen ein Computer eingesetzt wurde, 22 Kinder besuchten zwei Gruppen ohne Computereinsatz (Kontrollbedingung).

Die Autoren kommen nach Auswertung der Daten zu dem Schluss, dass die kognitive Leistungsfähigkeit der Kinder in den Gruppen mit Computereinsatz signifikant gesteigert werden konnte, während der Anstieg der kognitiven Leistungsfähigkeit in den beiden Kontrollgruppen unterhalb der Signifikanzschwelle blieb (Aufenanger \& Gerlach, 2008, S. 43 - 50). "Damit wird", so die Autoren "auf jeden Fall deutlich, dass der Computereinsatz [...] nicht nur keine negativen Folgen auf die kognitive und sprachliche Entwicklung der beteiligten Kinder hatte, sondern im Gegenteil sogar zu positiven Entwicklungsfortschritten führte" (S. 46). Bezüglich der Computerkompetenz stellen die Autoren fest, dass die Erhebungen zur Computerkompetenz der befragten Kinder eindeutig zeigten, dass in dem Projektjahr die vorhandenen Kompetenzen ausgeweitet wurden (S. 54). Da diese Aussage allein aufgrund eines Pre-Post-Vergleiches der computerbezogenen Fertigkeiten von Kindern in den Interventionsgruppen be-

125 (wenn man sie nicht gerade allein in ein dunkles, schallisoliertes Zimmer sperrt...) 
ruht, erscheint diese Aussage wenig überraschend, wie auch die Autoren selbst einräumen. Doch sie ergänzen: "Dass Kinder der Altersgruppe von drei bis sechs Jahren mit dem Computer so enorme Fortschritte machen können, dürfte doch überraschen" (ebenda). Da ein Vergleich mit Kindern aus Kontrollgruppen fehlt, wird letztlich nicht deutlich, wie die Autoren zu dieser Bewertung kommen.

Allgemein muss im Hinblick auf die Studie festgestellt werden, dass die Validierung ihrer Kernbefunde aufgrund zahlreicher Mängel in Forschungsdesign und Datenauswertung nicht gelingen kann. Neben einer stark an Kriterien der qualitativen Sozialforschung ausgerichteten Art der Stichprobenziehung (keine Zufallsauswahl, sondern bewusste Auswahl nach größtmöglicher Varianz der teilnehmenden Einrichtungen nach Region, Trägerschaft und Erfahrung mit Computereinsatz), in deren Rahmen die Zuweisung zu Kontroll- und Interventionsgruppe nicht zufällig erfolgte, ist auch das Verhältnis von sechs Interventionsgruppen $\mathrm{zu} 2$ Kontrollgruppen (von denen eine nur $\mathrm{n}=6$ Kinder aufwies) als problematisch anzusehen. Auch bei der Auswertung einer zentralen abhängigen Variable des Forschungsprojektes, der kognitiven Leistungsfähigkeit der Kinder mittels K-ABC, bleiben die Autoren den schlüssigen Beleg ihrer Folgerungen schuldig. Zwar verwenden sie im Rahmen ihrer Analyse altersnormierte Standardwerte (und werden so der Problematik gerecht, dass sich die kognitive Leistungsfähigkeit von Kindergartenkindern nach einem Jahr durchschnittlich immer erhöhen wird) und versuchen die Re-Test-Problematik bei Vorher-Nachher-Messungen durch Verwendung von Messwiederholungs-t-Tests einzugrenzen. Die große Heterogenität von Interventions- und Kontrollgruppe lässt jedoch keine validen Schlüsse zu, zumal Kontroll- und Interventionsgruppe nie direkt gegeneinander getestet werden (etwa im Rahmen einer Varianzanalyse mit Messwiederholung), sondern nur das Signifikanzniveau der Messwiederholungs-t-Tests in beiden Gruppen miteinander verglichen wird (wobei die nicht signifikanten Werte der Kontrollgruppe im Forschungsbericht nicht dokumentiert werden). Wenn also die Aussage, dass eine pädagogisch angeleitete Auseinandersetzung mit kindgerechter Edutainment-Software nicht zu negativen Entwicklungsfolgen führt, sachlogisch nicht in Zweifel gezogen werden soll, muss doch kritisiert werden, dass die Autoren vor dem Hintergrund der oben beschriebenen Mängel den Nachweis schuldig bleiben, der Einsatz von Computern im Kindergarten führe tatsächlich zu einer überdurchschnittlichen Entwicklung der kognitiven Leistungsfähigkeit von Kindergartenkindern.

Insgesamt zeigt sich, dass in der Literatur nur wenige - teilweise fragwürdig evaluierte Programme zur Förderung von Medienkompetenz zu finden sind, in deren Rahmen Medienkompetenzen vermittelt werden, die kaum dabei helfen 
können, die Frage zu beantworten, wie die Entstehung problematischer Mediennutzungsmuster wirkungsvoll verhindert werden kann.

\subsubsection{Medienkompetenz als Konzept zur Minderung problematischer Medien- nutzung - Fazit}

In diesem Kapitel wurden maßgebliche Konzeptionen von Medienkompetenz dargestellt, es wurden aktuelle Daten zur medienerzieherischen Praxis in Schulen und Kindergärten zusammengefasst und wissenschaftlich evaluierte Projekte zur Medienkompetenzförderung vorgestellt. Dabei konnte gezeigt werden, dass unter dem semantischen Dach des Begriffs „Medienkompetenz“ eine Reihe unterschiedlich konkreter Vorstellungen darüber versammelt ist, welche Medienkompetenzen Kinder, Jugendliche und Erwachsene haben oder aber haben sollten.

Es zeigte sich, dass weder die theoretischen Medienkompetenzkonzeptionen noch empirisch evaluierte Medienkompetenzprogramme gute Ansatzpunkte zur Entwicklung eines eigenen Medienkompetenzprogramms bilden, dessen Ziel es sein soll, die Entwicklung problematischer Mediennutzungsmuster in der Kindheit zu vermeiden. Als deutlich wertvoller erwiesen sich in dieser Hinsicht die Untersuchungen zur allgemeinen medienerzieherischen Kompetenz der pädagogischen Fachkräfte in Kindergärten und Schulen, sowie die Untersuchungen zur allgemeinen Verbreitung medienerzieherischer Maßnahmen in Kindergärten und Schulen. Hier zeigten sich deutliche Ansatzpunkte für eigene Bemühungen in der Fortbildung von Pädagogen und in der Entwicklung geeigneter Materialien.

Darüber hinaus wurde in der kritischen Auseinandersetzung mit einigen zentralen Aussagen der Medienkompetenzforschung gezeigt, dass die fehlende Anschlussfähigkeit theoretischer Medienkompetenzkonzepte für die Entwicklung eigener Bemühungen in der medienbezogenen Prävention durchaus systematisch begründet erscheint. Erstens wurde gezeigt, dass die aktuelle Literatur zur Entwicklung von Medienkompetenz entwicklungspsychologische Aspekte zu wenig berücksichtigt und daher die Besonderheiten einer kindlichen Zielgruppe zu wenig berücksichtigt werden können. Zweitens erscheint die Skepsis der Medienkompetenzforschung gegenüber Theorien und Erkenntnissen der Medienwirkungsforschung als zu gravierend, als dass sich die Autoren von Medienkompetenzkonzepten bereit sehen, diese Erkenntnisse anzuerkennen und in entsprechenden Konzepten zu berücksichtigen. Drittens wurde gezeigt, dass die Erwartungen, mithilfe von institutioneller Medienkompetenzförderung (unter 
Umständen problematische) Alltagsmedien-nutzungsmuster nachhaltig im positiven Sinn beeinflussen und verändern zu können, aus Sicht der Medienkompetenzforschung sehr gering sind. Viertens lässt sich festhalten, dass nur wenig und teilweise methodisch defizitäre systematisch-empirische Forschung zu konkreter institutioneller Medienkompetenzförderung im Kindesalter vorliegt, und dass die vorhandene Forschung sich auf, für den Fokus dieser Arbeit, deutlich periphere Bereiche der Medienkompetenzvermittlung bezieht (vgl. S. 173 179 in dieser Arbeit).

\subsubsection{Die Health-Promotion-Perspektive}

Die Bezeichnung Health-Promotion-Perspektive stellt, wie auf Seite 158 dieser Arbeit bereits angesprochen, eine recht grobe Vereinfachung verschiedener Forschungsdisziplinen und -perspektiven dar. So werden im Folgenden Forschungsansätze der Public-Health-Forschung/Gesundheitswissenschaft, der psychologischen Gesundheitsförderung, der Medizin (insbesondere der Pädiatrie) und Teile der Medienwissenschaft dargestellt ${ }^{126}$. Die Zusammenfassung dieser unterschiedlichen Perspektiven folgt der Definition von Haisch, Weitkunat und Wildner (1999, S. 317), die Public Health definieren als „Wissenschaft und Praxis der Krankheitsverhütung, Lebensverlängerung und Gesundheitsförderung durch organisierte, gemeindebezogene Maßnahmen“. Mediennutzung von Kindern und Jugendlichen ist aus dieser Perspektive heraus im Idealfall Teil eines gesunden Lebensstils und trägt - analog zum in der Verfassung der Weltgesundheitsorganisation (World Health Organisation, 2009, S. 1) definierten Gesundheitsbegriff - zum „,vollständigen körperlichen, seelischen und sozialen Wohlergehen“ eines Individuums bei, während Mediennutzung im schlimmsten Fall Auslöser und Verstärker problematischer oder pathologischer Entwicklungen sind kann. Die Wahrnehmung von Mediennutzung als Risikofaktor ist dabei insbesondere durch die Medienwirkungsforschung beeinflusst, deren Ergebnisse - trotz Kritik an methodischen Mängeln und unter Verweis auf weiteren Forschungsbedarf - beispielsweise von Meixner und Jerusalem als deutlicher Indikator für mediennutzungsbezogenen Prä-

126 Zur Diskussion über Verflechtungen und Abgrenzungskriterien zwischen Gesundheitswissenschaft/Public-Health-Forschung und Gesundheitspsychologie siehe Weitkunat, Haisch und Kessler (1997); zur Abgrenzung zwischen Public-Health-Ansätzen und Medizin vgl. Hurrelmann, K., Laaser \& Razum (2006, S. 23); zum Beitrag medienwissenschaftlicher Forschung für die Public-Health-Forschung vgl. Jazbinsek (2001). 
ventionsbedarf im Hinblick auf die Gesundheitsförderung von Kindern und Jugendlichen bewertet wird (Meixner \& Jerusalem, 2006, S. 219). Kline fasst die Health-Promotion-Perspektive auf Mediennutzung als potentiellen Risikofaktor unter Berufung auf Berichte des United States Public Service (The Surgeon General's Vision for a Healthy and Fit Nation, 2010, S. 6 - 7) folgendermaßen zusammen:

"From a public health point of view a risk factor is anything that increases the probability that a person will suffer harm. [...] concepts of risk and protection' can be central in framing a 'public health' approach to media which 'involves identifying risk and protective factors, determining how they work, making the public aware of these findings and designing programs to prevent or stop them. (Kline, 2005, S. 240).

Die Forderung nach einer Health-Promotion-Perspektive auf kindliche und jugendliche Mediennutzung wurde bisher nur in Ansätzen aufgenommen. Einer dieser Ansätze bezieht sich auf die Prävention von Übergewichtigkeit. Werbefinanzierte Unterhaltungsmedien wurden frühzeitig als einer der Faktoren identifiziert, der zur Übergewichtigkeit der Bevölkerung beiträgt, obgleich die von der Medienwirkungsforschung gefundenen Effekte als schwach zu bezeichnen sind (vgl. S. 109 - 113 in dieser Arbeit). Ebenso wird bereits seit Längerem die Rolle der Medien bei der Entstehung von Essstörungen diskutiert und erforscht (vgl. S. $113-115$ in dieser Arbeit). Auch von Seiten der Medienwissenschaft wurde bereits eine Reihe von Studien vorgelegt, die sich mit der Frage beschäftigen, mithilfe welcher Instrumente die Effekte der Gewaltmediennutzung minimiert werden können (vgl. zum Beispiel Studien zu wirksamen elterlichen Interventionsstrategien auf S. 125 - 130 in dieser Arbeit).

Eine umfassende Integration problematischer Mediennutzung im Sinne der in Kapitel 6 geleisteten Definition spielt in gesundheitswissenschaftlichen Überlegungen zur Gesundheitsförderung von Kindern und Jugendlichen bisher jedoch eine untergeordnete Rolle ${ }^{127}$. Dennoch existiert, im Gegensatz zu Programmen

127 Es wurde mit Bezug auf die Gewaltmedienwirkungsforschung vereinzelt sogar davor gewarnt, die Prävention von Gewaltmediennutzung zum Gegenstand der Gesundheitsförderung zu machen, bevor nicht die genauen Prozesse der Wirkungen von Mediengewalt auf Aggressivität geklärt worden sind (The Lancet, 2008). So sei die USamerikanische Wirkungsforschung, auf die auch im Rahmen dieser Arbeit maßgeblich Bezug genommen wird (vgl. vor allem Anderson, C. A. et al., 2008; Bushman \& Huesmann, 2006; Gentile et al., 2004), inzwischen stark politisiert und dementsprechend interessengeleitet: „In the USA, tackling violence is highly politicised. Most of the media violence research is led by a group of prominent US psychologists who have helped influence the health agendas of the American Medical Association and American Association of Pediatrics." (S. 1137). Erst wenn noch weitere Aspekte der Gewaltmedienwirkung erforscht seien, solle über entsprechende Präventionsmaßnahmen entschieden werden: „With these kind of data we can establish whether media violence may or may not exac- 
der Medienkompetenzförderung, eine ganze Reihe evaluierter Programme zur Förderung eines adäquaten Medienumgangs, die im nachfolgenden Kapitel noch eingehender beschrieben werden (vgl. S. 185 - 189 in dieser Arbeit). Vor allem hat insbesondere die Forschung zur Gesundheitsförderung von Kindern wichtige Arbeit dahingehend geleistet, Gesundheitsförderungsprogramme theoretisch zu fundieren, im Hinblick auf bestimmte Zielgruppen $\mathrm{zu}$ optimieren und $\mathrm{zu}$ erforschen, welche Akteure und Institutionen in besonderer Weise geeignet sind, erfolgreiche Gesundheitsförderungsprogramme zu gestalten.

\subsubsection{Zur Theorie von Intervention und Prävention}

Die erste grundlegende Definition und Unterteilung präventiver Maßnahmen lieferte Caplan, der Präventionsmaßnahmen in primäre, sekundäre und tertiäre Prävention unterteilte (Caplan, 1964). War Caplans Systematisierung damit zunächst auf psychologische beziehungsweise psychiatrische Anwendungsfelder gerichtet, hat sie inzwischen in verschiedensten sozialen und humanwissenschaftlichen Bereichen breite Akzeptanz gefunden ${ }^{128}$. Eine primäre Prävention nach Caplan hat das Ziel der Senkung der Inzidenzraten psychischer Störungen und Gesundheitsgefährdungen in umschriebenen Populationen (Rollett, 2002, S. 715). Allgemeiner formuliert geht es bei primären Präventionen um die Verringerung des Neuauftretens als negativ wahrgenommener Handlungen oder als negativ wahrgenommener Zustände von Individuen insbesondere durch die Eliminierung oder Reduzierung von Risikofaktoren. Bemühungen, durch frühzeitige Medienerziehung medienbezogenes Risikoverhalten zu verhindern, fallen somit in den Bereich der Primärprävention. Als sekundäre Prävention be-

erbate existing mental, psychological, or behavioural health problems. Only then should research focus on how changes in media use can reduce those risks, and not before" (ebenda). Vertreter dieser Ansicht - so wie der Editor des Lancet - verkennen allerdings die Tatsache, dass im Hinblick auf die Gewaltmedienwirkungsforschung mit Sicherheit noch sehr viele Jahre vergehen werden, bis sich die Mitglieder der Scientific Community auf ein konkretes Gewaltmedienwirkungsmodell geeinigt haben. Darüber hinaus ist nicht erkennbar, dass in der Scientic Community tatsächlich Zweifel am Einfluss von Gewaltmediennutzung zumindest auf kindliches Verhalten bestehen.

$128 \mathrm{Da}$ in der kommunikationswissenschaftlichen wie auch der pädagogischen Forschung entsprechende grundlegende Systematisierungen fehlen, wird im Folgenden fast ausschließlich auf Konzepte aus dem Bereich der Public- Health- wie der psychologischen Gesundheitsforschung zurückgegriffen. Es sei aber grundsätzlich darauf verwiesen, dass damit problematische Mediennutzungsmuster nicht per se mit einer Auffälligkeit bzw. Störung in einem psychologisch-medizinischen Verständnis gleichgesetzt werden. 
zeichnete Caplan die Senkung der Prävalenzraten von Krankheiten dank Früherkennung (Screening) und frühzeitiger Behandlung (Caplan, 1964). Übertragen in den subklinischen Bereich bedeutet dies, dass bereits aufgetretene, aber beeinflussbare Fehlentwicklungen korrigiert werden sollen, um weitere negative Konsequenzen zu verhindern (Rollett, 2002, S. 716). Bezogen auf die Prävention medienbezogenen Risikoverhaltens heißt dies, dass bereits bestehende problematische Mediennutzungsmuster korrigiert und bereits eingetretenen negativen Effekten frühzeitig begegnet werden soll. Tertiäre Prävention schließlich hat nach Caplan das Ziel, auf bereits eingetretene Schädigungen einzuwirken (ebenda) und deckt sich damit weitgehend mit dem Konzept der Rehabilitation. Bezogen auf problematische Mediennutzungsmuster existieren tertiärpräventive Einrichtungen im Bereich der Nachsorge von Computerspielabhängigkeit.

Medienpräventionsprogramme, die in der Regel darauf zielen, negative Medienwirkungen im Vorhinein zu vermeiden, sind somit dem Bereich der Primärprävention zuzuordnen, wobei in der Regel sekundärpräventive Elemente (Identifikation bereits vorhandener problematischer Mediennutzungsmuster und gegebenenfalls Minderung negativer Effekte) enthalten sind. Dabei lässt sich auch die Primärprävention noch weiter untergliedern in universelle Präventionsprogramme, die eine gesamte Population ansprechen und selektive Programme, die Teile eine Population ansprechen, die durch das Vorhandensein bestimmter bekannter Risikofaktoren risikodisponierter erscheinen als die Durchschnittspopulation (Cardemil \& Barber, 2001). Jenseits der medizinischen Prävention definiert Franzkowiak (2010) zwei Zielrichtungen von Prävention: Die Verhaltensprävention, die auf die Änderung als risikobehaftet identifizierter Verhaltensweisen zielt und die Verhältnisprävention, die auf die Änderung als risikobehaftet geltender Umwelt- und Lebensbedingungen fokussiert ist. Eine systemische Perspektive der Prävention versucht, Instrumente beider Ansätze zur verknüpfen.

Jerusalem unterscheidet weiterhin zwischen pathogenetisch beziehungsweise risikoorientierter Prävention und ressourcenorientierter Prävention. Risikoorientierte Präventionsprogramme zur Gesundheitsförderung im Kindes- und Jugendalter bestehen demnach darin, „gesundheitliche Risiken und Risikoverhaltensweisen zu vermeiden oder, wenn dies nicht gelingt, ihre gesundheitsschädlichen Folgen möglichst gering zu halten“ (Jerusalem, 2006, S. 32). Im Gegensatz dazu zielt ressourcenorientierte Gesundheitsförderung ,auf die Stärkung individueller Ressourcen (zum Beispiel emotionale, kognitive und soziale Kompetenzen) oder systemischer Ressourcen (zum Beispiel Familienzusammenhalt, Schulklima)“ (Jerusalem, 2006, S. 33). Es lassen sich bei ressourcenorientierter Gesundheitsförderung darüber hinaus themenspezifische 
Programme, die bestimmte Fähigkeiten beziehungsweise Kompetenzen stärken sollen, von unspezifischen Programmen unterscheiden, die beispielsweise allgemeine Lebenskompetenzen fördern sollen (ebenda).

Besondere Aufmerksamkeit wird in der Präventionsforschung der Frage gewidmet, welche Faktoren dazu beitragen, ein Präventionskonzept erfolgreich zu gestalten. Bond und Hauf (2004) haben auf der Grundlage einer Sichtung der Literatur zur erfolgreichen Primärprävention psychischer Gesundheitsstörungen einen Katalog der zehn wichtigsten Erfolgsfaktoren primärpräventiver Maßnahmen vorgelegt, der sich auch auf primärpräventive Maßnahmen in anderen Anwendungsbereichen übertragen lässt:

(1) Theorie- und Forschungsbasierung bei Entwicklung der Maßnahmeninhalte, der Maßnahmenstruktur und der Implementation.

(2) Eindeutige und realistische Zielformulierung, die bei den beteiligten Gruppen auf breite Zustimmung stößt.

(3) System- und ebenenübergreifende Perspektive mit Berücksichtigung vielfältiger Einflussmöglichkeiten auf die Entwicklung und vielfältiger Entwicklungswege.

(4) Angemessene Dosierung der Maßnahme mit Berücksichtigung von Erinnerungen an die Maßnahmeninhalte in gewissen Zeitabständen („,booster sessions", ,follow ups"), um die erwünschten Ergebnisse zu erreichen und aufrechtzuerhalten.

(5) Berücksichtigung der vorhandenen Stärken, Kompetenzen und protektiven Faktoren, aber auch der Risiken und Schwierigkeiten beteiligter Individuen und Systeme.

(6) Sensitivität für die besonderen Belange der Zielgruppe bei den Maßnahmeinhalten, der Maßnahmenstruktur und der Implementation.

(7) Qualitativ anspruchsvolle Evaluation (mit Berücksichtigung auch unerwünschter Effekte) und laufende Überwachung der Maßnahmenumsetzung.

(8) Umfangreiche Maßnahmendokumentation zur Nutzbarmachung in verschiedenen Kontexten und durch verschiedene Vermittler.

(9) Berücksichtigung der Ressourcen, die zur Maßnahmenumsetzung erforderlich sind.

(10) Berücksichtigung des sozio-politischen Kontextes, in dem die Maßnahme zum Einsatz kommen soll.

(Bond \& Hauf, 2004; deutsche Übersetzung angelehnt an Lohaus, Domsch \& Klein-Heßling, 2008, S. 509 - 510) 
Im Hinblick auf die Frage, welche Faktoren bei Präventionsprogrammen im Kindesalter besonders zu berücksichtigen sind, werden in der Literatur vornehmlich die folgenden Punkte diskutiert: Alter der Kinder, Programminhalte, die beteiligten Akteure sowie der institutionelle Kontext des Programms. Lohaus und Lißmann bemerken allerdings, dass eine umfassende theoretische Konzeption der Prävention im Kindesalter, die insbesondere entwicklungspsychologische Aspekte berücksichtigt, bisher fehlt. Sie verweisen jedoch gleichzeitig auf eine Fülle evaluierter erfolgreicher Einzelmaßnahmen (2006, S. 84), deren konzeptionelle Vorannahmen Vorbildcharakter für weitere Programme haben könnten. Vor diesem Hintergrund sollen nachfolgend die wichtigsten derzeit bekannten Präventionsprogramme dargestellt werden, die einen veränderten Umgang mit Medien oder eine stärkere Reflexion des Medienumgangs Minderjähriger zum Ziel hatten. Anschließend sollen daraus Implikationen und zentrale Erfolgsfaktoren für die Konzeption eines eigenen Präventionsprogramms abgeleitet werden.

\subsubsection{Empirische Ergebnisse}

Bezüglich bereits durchgeführter medienbezogener Präventionsprogramme lassen sich zwei Hauptgruppen medienbezogener Aktivitäten unterscheiden, in denen positive Interventionseffekte nachgewiesen werden konnten ${ }^{129}$ : Präventionsprogramme zur Gewaltreduktion sowie Programme zur Förderung gesunden Ernährungs- und Bewegungsverhaltens ${ }^{130}$. Die Prävention beziehungsweise Reflexion problematischer Mediennutzungsmuster ist somit in diesen Programmen immer Mittel zur Erreichung anderer gesundheitsbezogener

129 Nachfolgend wird nur auf solche Interventionsprogramme eingegangen, zu denen eine wissenschaftlich-evaluative und veröffentlichte Begleitforschung vorliegt. Interventionsprogramme ohne Begleitforschung bzw. mit lediglich anhand "Grauer Literatur" belegter Begleitforschung werden nicht berücksichtigt. Zudem beschränkt sich die nachfolgende Darstellung auf solche Studien, deren Hauptzielgruppe Kinder oder Jugendliche darstellen.

130 Programme zur Prävention von Tabakkonsum beinhalten teilweise zwar ebenfalls Elemente der Reflexion der eigenen Mediennutzung (insbesondere mit Bezug auf Werbeinhalte und ihre Wirkung; vgl. hier Gonzales, Glik, Davoudi \& Ang, 2004; Pinkleton, Weintraub Austin, Cohen, Miller \& Fitzgerald, 2007), werden aufgrund des insgesamt geringen Mediennutzungsbezugs an dieser Stelle ausgeklammert, zumal sich die gefundenen Programme allein auf Jugendliche beziehen und lediglich Effekte auf der Einstellungsebene, nicht aber auf der Verhaltensebene zeigten. 
beziehungsweise sozialer Ziele. All diesen Maßnahmen ist gemein, dass sie fast ausschließlich auf Fernsehnutzungsgewohnheiten von Kindern oder Jugendlichen zielen und bei der Reflexion von Medieninhalten wiederum fast ausschließlich Fernsehbotschaften gemeint sind, während andere Formen elektronischer Mediennutzung kaum eine Rolle spielen.

\subsection{Mediengewaltprävention}

Kunczik und Zipfel unterscheiden in ihrem Überblick zu Interventionen (hier im Sinne primär- oder sekundärpräventiver Präventionsmaßahmen zu verstehen) zum Umgang mit Mediengewalt unter Bezug auf Cantor und Wilson (Cantor \& Wilson, 2003) drei grundsätzlich verschiedene Interventionsstrategien und trennen die unterschiedlichen Interventionsmaßnahmen nach den Hauptakteuren bei der Durchführung einer Maßnahme (Kunczik, M. \& Zipfel, 2004). Unter „Interventionsstrategien“ verstehen die Autoren dabei sowohl Maßnahmen zur Förderung der Medienkompetenz im Sinne eines kritischen Verständnisses von Medieninhalten, als auch restriktive Maßnahmen, die beispielsweise die Rezeption problematischer Inhalte (beziehungsweise eine Reduktion des Fernsehkonsums) verhindern. Als erste Untergruppe von Medieninterventionsstrategien nennen Kunczik und Zipfel Maßnahmen, die von Eltern ergriffen werden. Die zweite Gruppe umfasst medienpädagogische Lehrpläne und Programme an Schulen, die dritte Gruppe subsumiert (massen-) medienvermittelte Botschaften (Kunczik, M. \& Zipfel, 2004).

Mit Hinblick auf Forschungsergebnisse $\mathrm{zu}$ elterlichen Interventionsmaßnahmen kommen die Autoren dabei zu dem Schluss, dass solche Maßnahmen generell effektiv sein können, wenn Eltern aktive Interventionsstrategien anwenden (Eltern sprechen mit Kindern über Medieninhalte und bewerten diese Medieninhalte explizit) und - bei jüngeren Kindern - klare Regeln und Restriktionen formulieren (vgl. auch Kapitel 5, S. 125 - 130 in dieser Arbeit). Je jünger die Kinder sind, desto erfolgversprechender seien die Maßnahmen (Kunczik, M. \& Zipfel, 2004). Allerdings verweisen die Autoren unter Bezug auf Studien von Valkenburg, Warren und anderen darauf, dass ein hoher Bildungsstand der Eltern, eine kontinuierliche Anwesenheit der Eltern zu Hause, eine generelle Besorgnis der Eltern über negative Medieneffekte und ein generell hohes elterliches Engagement die Anwendung medienpädagogischer Maßnahmen fördern (Kunczik \& Zipfel, 2004; Valkenburg, Krcmar, Peeters \& Marseille, 1999; Warren, 2001; Warren, Gerke \& Kelly, 2002) und somit im 
Umkehrschluss besonders die Kinder durch solche Maßnahmen nicht erreicht werden, die ihrer am Dringendsten bedürften.

Insofern wächst nach Kunczik und Zipfel die Bedeutung schulischer Interventionsprogramme, deren Ergebnisse sie wie folgt zusammenfassen: Obwohl nur wenige wissenschaftlich evaluierte Schulinterventionsprogramme bezüglich der Reduktion und Reflexion von Mediengewaltinhalten vorliegen (Cantor \& Wilson, 2003; Rosenkoetter, Rosenkoetter, Ozretich \& Acock, 2004), kann insgesamt ein Erfolg schulischer Programme zur Medienerziehung konstatiert werden, wenn entsprechende Maßnahmen über einen längeren Zeitraum wiederholt werden. Dabei haben Programme, in deren Rahmen die Reflexion über schädliche Wirkungen von Mediengewalt im Mittelpunkt steht, zwar eine Wirkung bezüglich der Reduktion von Mediengewalteffekten (zum Beispiel Reduktion von Gewaltverhalten), die Faszination gegenüber Mediengewalt sowie eine deutlich geringe Nutzung von Mediengewalt konnte jedoch nicht erreicht beziehungsweise belegt werden (Kunczik, M. \& Zipfel, 2004; Rosenkoetter et al., 2004).

Interessanterweise sind Maßnahmen, die allein auf die Drosselung von Medienkonsum zielen, ohne dabei das Thema "Gewalt" direkt zu thematisieren ebenfalls in der Lage, Effekte von Mediengewalt zu reduzieren (Robinson, Wilde, Navracruz, Haydel \& Varady, 2001). Diese Ergebnisse könnten derart interpretiert werden, dass gut gemachte Programme mit Elementen, in denen Kinder intensiv über Mediengewalt reflektieren, zwar kurzfristig in der Lage sind, Gewaltverhalten zu reduzieren ${ }^{131}$, dass langfristig aber keine Wirkungen erzielt werden können, da das Mediennutzungsverhalten selbst nicht wesentlich beeinflusst werden konnte. In Verbindung mit dem von Kunczik und Zipfel berichteten Resultat, dass Reflexionselemente vor allem bei älteren Schülergruppen erfolgreich sind, muss befürchtet werden, dass schulische Interventionsprogramme für jüngere Schüler, in denen reflexive Elemente vor verhaltensnäheren Elementen ${ }^{132}$ dominieren, nicht oder nur kurzfristig erfolgreich sind ${ }^{133}$. Vielmehr sollten schulische Medieninterventionselemente für

131 Diese Interpretation liegt auch nahe, da Gewaltverhalten innerhalb der Studien oft in Form von schulischer Gewalt gemessen wurde, beispielsweise über ein Peer-RatingVerfahren, in dem Schüler/innen einer Klasse alle anderen Schüler/innen ihrer Klasse bezüglich ihres Gewaltverhaltens einstufen müssen. Dass didaktisch gut aufgebaute und interessante Inhalte in der Lage sind, das Sozialverhalten von Schüler/innen beispielsweise auf dem Schulhof kurzfristig positiv zu beeinflussen, liegt auf der Hand.

132 Zum Beispiel die Vereinbarung eines bestimmten Mediennutzungsverhaltens über einen definierten Zeitraum.

133 Kunczik und Zipfel verweisen sogar auf eine Studie von Doolittle, in der gezeigt wurde, dass Schulprogramme kontraproduktiv sind, wenn Schüler/innen zur Produktion gewalt- 
jüngere Zielgruppen auch immer Regeln hinsichtlich angemessener Mediennutzungszeiten und angemessener Medieninhalte vermitteln und entsprechendes Verhalten im Klassenverband einüben. Robinson und Kollegen (1999; 2001) beziehen sich bei der Beschreibung der Unterrichtsinhalte explizit auf die Erkenntnisse der sozial-kognitiven Lerntheorie Banduras (1979), in der Lernen durch die Orientierung an Verhaltensmodellen im Mittelpunkt steht.

Eine dritte Gruppe präventiver Maßnahmen fassen Kunczik und Zipfel unter dem Punkt "Medieninhalte mit Anti-Gewalt-Botschaften" zusammen, wobei sie sich im Wesentlichen auf eine Analyse von Cantor und Wilson stützen (Cantor \& Wilson, 2003). Insgesamt, so die Autoren, können solche Programme durchaus positive Effekte erzielen, wenn die Programme sehr eindeutige Botschaften enthalten und im Rahmen der Thematisierung von Gewalt vor allem das Leiden der Opfer darstellen (Kunczik, M. \& Zipfel, 2004). Allerdings konnten die analysierten Studien in der Regel lediglich Einstellungsänderungen bezüglich Gewalt beziehungsweise eine höhere Empathie der Rezipienten bezüglich des Leidens der Opfer messen, eine tatsächliche Reduktion von Gewaltverhalten wurde nicht berichtet.

\subsubsection{2 Übergewichtsprävention}

Eine bedeutende Zahl in der Vergangenheit durchgeführter Präventionsstudien ging der Frage nach, in welchem Maß medienerzieherische Maßnahmen geeignet sind, Gewichtsprobleme von Kindern und Jugendlichen zu reduzieren und die körperliche Fitness zu verbessern. Ausgehend von Studien zum Zusammenhang zwischen Medienkonsum und Gewichtsproblemen (Adipositas beziehungsweise Anorexie), besteht der Ansatz dieser Studien darin, Kinder und Jugendliche zu einem maßvollen Umgang mit den Medien zu motivieren und zugleich den Einfluss von Werbung und medialen Stereotypen auf das eigene Ernährungsverhalten zu erkennen. Auch hier lassen sich Studien zur Effektivität elterlicher Maßnahmen und Studien zur Effektivität schulischer Programme unterscheiden.

Epstein und andere konnten in einer zweijährigen feldexperimentellen Studie nachweisen, dass sich Medienzeiten (Fernsehzeiten und Computerspielzeiten) von durchschnittlich sechsjährigen Kindern signifikant und dauerhaft verringern lassen, wenn Eltern ein elektronisches Gerät zur Regulierung der Medienzeiten

haltiger Medien angeregt werden, um ihr allgemeines Involvement zu erhöhen (Doolittle, 1980; Kunczik, M. \& Zipfel, 2004). Es ist allerdings fraglich, inwieweit dies noch mit einer Reflexion über Mediengewalt beschrieben werden kann. 
ihrer Kinder einsetzen (behavioral engineering technology) (Epstein et al., 2008). Gleichzeitig wurde in dieser Studie auch nachgewiesen, dass sich in der Interventionsgruppe Ernährungsverhalten und Body-Mass-Index der Probanden deutlich günstiger entwickelte als in der Kontrollgruppe. Allerdings berichtet Epstein von einer hohen Verweigerungs- beziehungsweise Abbruchquote unter eingangs kontaktierten Familien. Von 185 kontaktierten Familien nahmen 115 nicht an der Untersuchung teil beziehungsweise brachen die Studie ab, wobei unter 51 dieser Fälle systematische Ausfälle befürchtet werden müssen, da bei Kindern dieser Familien der Body-Mass-Index offenbar oberhalb des 75. Perzentils lag und auch erhebliche Medienzeiten berichtet wurden, sie mithin zur Kernzielgruppe der Studie gehörten.

Insofern erscheinen auch hier insbesondere Studien zur Effektivität schulischer Maßnahmen interessant, da sich über die Schulen eine breitere Bevölkerungsgruppe erreichen lässt. Tatsächlich konnten Robinson und andere mithilfe eines ähnlichen Studiendesigns wie bei Epstein zeigen, dass ähnliche Effekte auch erzielt werden können, wenn eine behavioral engineering technology in Kombination mit schulischem Medienunterricht in der Grundschule eingesetzt wird (Robinson, 1999), ohne dass dabei ähnliche hohe Abbruchraten berichtet wurden ${ }^{134}$. Aber auch ohne den Einsatz elektronischer Medienzeitbegrenzer konnten Austin und andere zeigen, dass Medienunterricht ein effektives Mittel ist, gestörte Ernährungsgewohnheiten (Adipositas wie auch Anorexie) zu verändern (Austin, Field, Wiecha, Peterson \& Gortmaker, 2005). Dabei war diese Intervention, die sich ausschließlich auf Mädchen sechster und siebter Schulklassen bezog, aber nicht allein medien- beziehungsweise mediennutzungsbezogen, sondern integrierte die medienbezogene Intervention in ein Bündel weiterer Maßnahmen (Aufklärung über Ernährungsfragen im Unterricht etc.), so dass es schwierig ist, eine Aussage darüber zu treffen, in welcher Weise der medienbezogene Teil des Programms zum Erfolg des Gesamtprogramms beigetragen hat. So konnten Salmon und andere zeigen, dass auch ein Ernährungsunterrichtsprogramm ohne Medienbezug geeignet sein kann, Mediennutzung zu reduzieren (Salmon, J., Ball, Hume, Booth \& Crawford, 2008).

134 Robinson setze dabei auf einen reinen Medienunterricht, Informationen über gesunde Ernährung etc. wurden den Schülern nicht gegeben. 
